\title{
Proceedings
}

\section{Abstracts of the Falls Frailty Bone Health 2017}

\author{
$9^{\text {th }}=10^{\text {th }}$ November 2017 in Dublin, Ireland \\ Organizers: Professor Tahir Masud \& Professor Dawn Skelton
}

All published work is licensed under Creative Common License CC BY-NC-SA 4.O (Attribution-NonCommercial-ShareAlike)

\section{ONE-YEAR ADHERENCE TO A FALL PREVENTIVE EXERCISE PROGRAMME WITH OR WITHOUT BEHAVIOUR CHANGE SUPPORT IN COMMUNITY- DWELLING OLDER PEOPLE}

\begin{abstract}
Arkkukangas M. ${ }^{1}$, Söderlund A. ${ }^{2}$, Eriksson S. ${ }^{3,4,5}$ and Johansson A. ${ }^{2,6}$

'Research and Development Department in the Community of Eskilstuna, Sweden; ${ }^{2}$ School of Health, Care and Social Welfare, Mälardalen University, Box 883, SE-721 23 Västerås, Sweden; ${ }^{3}$ Centre for Clinical Research Sörmland, Uppsala University, Kungsgatan 41, 63188 Eskilstuna, Sweden; ${ }^{4}$ Department of Community Medicine and Rehabilitation, Physiotherapy, Umeå University, SE-901 87 Umeå, Sweden; ${ }^{5}$ Department of Neuroscience, Physiotherapy, Uppsala University, Box 593, BMC, Uppsala, Sweden; ${ }^{6}$ Centre for Clinical Research, Uppsala University, Västmanland County Hospital, 72189 Västerås, Sweden
\end{abstract}

Introduction: A sedentary lifestyle in older individuals is a threat to their future health and one of the leading risk factor for mortality. Home based exercise have been recommended in falls prevention however the effects on adherence or attendance for exercise have been sparsely studied and is both a prerequisite and a challenge for positive health outcomes. Therefore the aim was to study behavioural factors important for long-term exercise adherence to a fall preventive exercise among community-dwelling older persons.

Methodology: A total of 114 community dwelling older persons over 75 years of age participated in one of two groups based on; Otago Exercise Programme, OEP $(n=58)$ or the OEP combined with motivational interviewing, $\mathrm{MI}$ $(O E P+M I)(n=56)$. Exercise were followed up by exercise diaries reported by the older persons during one-year of exercising. Behaviour measurements at baseline were Readiness to Change, Falls self- efficacy and activity level.

Results: Exercising two times per week were accomplished by $47 \%$ in the OEP group and $64 \%$ in the OEP+MI group. The behavioral factors; activity habits at baseline and exercise supported by Ml positively predicted exercise adherence at 52 weeks follow up, odds ratio $3.39(95 \% \mathrm{Cl}, 1.38-8.32)$ respectively 2.47 (95\% $\mathrm{Cl}, 1.11-5.49)$.

Conclusions: Including the behaviour aspects; physical activity habits and exercise in combination with MI positively predicted exercise adherence and are behavioural factors to be considered when striving for fall preventive exercise adherence. Older persons with low activity habits would probably benefit from behaviour change support to overcome barriers for performing fall preventive exercise.

\section{THE RELATIONSHIP BETWEEN A SELF-REPORTED AND INFORMANT-RATED MEASUREMENT OF ACTIVITIES OF DAILY LIVING IN PEOPLE WITH COGNITIVE IMPAIRMENT}

Burgon C. ${ }^{1}$, van der Wardt V. ${ }^{1}$, Goldberg S. ${ }^{2}$ and Harwood R.H. ${ }^{1,3}$

'Division of Rehabilitation and Ageing, School of Medicine, University of Nottingham; '2School of Health Sciences, University of Nottingham; ${ }^{3}$ Health Care of Older People, Nottingham University Hospitals NHS Trust, Nottingham, UK

Introduction: Assessment of Activities of Daily Living (ADL) is recommended for falls risk assessment, and is an important outcome in frailty. Given the association between falls and cognitive impairment, and frailty and dementia, measurement of $A D L$ in people with cognitive impairment is of clinical relevance. This typically relies on caregiver proxy ratings. However, this method excludes the views of the person experiencing cognitive and functional changes, and some people requiring $A D L$ assessment do not have a suitable informant. This study sought to examine the feasibility of using self-reported ADL assessment, by examining its relationship with a popular informant-rated scale.

Methodology: Our study formed part of the larger feasibility trial: Promoting Activity, Independence and Stability in Early Dementia (PrAISED). People with Mild Cognitive Impairment and mild dementia were recruited to the study, along with an informant where possible. ADL was assessed using the self-reported Nottingham Extended Activities of Daily Living (NEADL) scale, originally validated in a stroke population, and the informant rated Disability Assessment for Dementia (DAD). The relationship between the self-reported and 
informant-rated ADL was explored through hierarchical linear regression.

Results: The analysis included 53 participants with an average age of 75 , and a mean standardized Mini Mental State Examination score of 25.6. 58.5\% were male. Both DAD scores $(M=77.9)$ and NEADL scores $(M=16.2)$ showed ceiling effects (DAD: 20.8\%; NEADL: 5.7\%). NEADL scores were moderately associated with DAD scores when controlling for sex, age, education and frailty $\left(R^{2}=.38\right.$; Beta $=.67 ; p<.001)$. None of the covariates were significant contributors to the model.

Conclusions: The moderately strong relationship between the NEADL and DAD scores indicates that the scales measure similar, but not identical, constructs. Both scales would benefit from better discrimination at the top end of the scores when used in $\mathrm{MCl}$ and mild dementia populations.

\section{EXPLORING THE PERSPECTIVES AND EXPERIENCES OF HEALTHCARE PROFESSIONALS ON PROVIDING FALLS INTERVENTIONS TO PEOPLE WITH DEMENTIA}

\begin{abstract}
Burgon C. ${ }^{1,2}$, Darby J. ${ }^{1}$, Pollock K. ${ }^{2}$, van der Wardt V. ${ }^{1}$, Peach T. ${ }^{3}$, Beck L. ${ }^{4}$, Logan P. ${ }^{1}$, Harwood R.H. ${ }^{1,5}$

'Division of Rehabilitation and Ageing, School of Medicine, University of Nottingham, Nottingham, United Kingdom; ${ }^{2}$ School of Health Sciences, University of Nottingham, Nottingham, United Kingdom; ${ }^{3}$ Nottingham CityCare Partnership, Nottingham, United Kingdom; ${ }^{4}$ Nottinghamshire Healthcare NHS Foundation Trust, Nottingham, United Kingdom; ${ }^{5}$ Health Care of Older People, Nottingham University Hospitals NHS Trust, Nottingham, United Kingdom
\end{abstract}

Introduction: Falls are more likely to occur in people with dementia, and whilst multifactorial interventions reduce risk of falls in older people, their effectiveness in people with dementia is uncertain. Falls interventions tailored to the needs of people with dementia are required. Understanding the experiences and perspectives of healthcare professionals (HCPs) currently providing interventions to this population can inform the development of falls interventions for people with dementia.

Methodology: This qualitative study used semi-structured interviews to explore HCPs' perspectives and experiences of providing falls interventions for people with dementia. The participants were nurses, occupational therapists, physiotherapists and doctors working in falls $(\mathrm{N}=11)$ or memory assessment services $(\mathrm{N}=8)$ in the United Kingdom. Interviews were transcribed verbatim and analysed through thematic analysis.

Results: The three main themes that emerged were: challenges posed by cognitive impairment, segregated service provision, and recommended adjustments. The HCPs identified that providing interventions was challenging due to patients' increased likelihood of falls, their difficulties with recall and insight, and sometimes their unwillingness to discuss falls risk due to stigma. The additional support required for these patients was difficult to provide due to limited resources. The memory and falls services were distinct and sometimes struggled to communicate effectively. Recommended adjustments to current falls prevention strategies included: regular and ongoing support; early stage intervention; support from a multidisciplinary team; and tailoring to each individual's needs, interests and preferences.

Conclusions: HCPs experience various challenges to providing interventions to people with dementia. Better integration of services and long term service provision is required to meet the mental and physical health needs of this population. Importantly, interventions should be tailored to the interests and preferences, as well as the needs, of each individual. Future research will be needed to test the effectiveness of these adapted interventions in people with dementia.

\section{THE PRAISED FEASIBILITY STUDY:}

\section{THE TIMED UP AND GO / DUAL TASK TIMED UP} AND GO DIFFERENCE COMPARED WITH SMMSE AND CANTAB TASKS

Lock J., Burgon C., van der Wardt V., Goldberg S., HoodMoore V., Booth V. and Harwood R.H.

University of Nottingham, School of Medicine, Division of Rehabilitation and Ageing

Introduction: People with dementia and Mild Cognitive Impairment $(\mathrm{MCl})$ are at high risk of falling with at least a twofold increased risk of falls compared with cognitively intact older people. Falls risk and mobility are often assessed with the Timed up and Go (TUG) and the Dual Task Timed Up and Go (D-TUG). This study will investigate the relationship between cognitive abilities and the additional cognitive challenge when performing the D-TUG.

Methodology: As part of the Promoting Independence, Activity and Stability in Early Dementia feasibility study (PrAISED), we assessed cognitive abilities using standardised Mini Mental State Examination (SMMSE) and the CANTAB computerised test battery, which included the Paired Associates Learning test (PAL), Spatial working Memory test (SWM) and Attention switching task (AST RL). The TUG was performed with and without dual task. The D-TUG included counting backwards in threes from a random number chosen by researchers between 60 and 100 .

Results: 60 participants ( $n=26$ Females, age range 65-91 years) with mild dementia or mild cognitive impairment (mean SMMSE=25.8, range 19-30) were recruited. Pearson's correlations showed that there was no significant relationship between cognition and the reduction in walking speed while dual tasking except for 
scores on a spatial working memory strategy score $(r=$ $-.31, \mathrm{n}=46, \mathrm{p}<0.05$ ).

Conclusion: The spatial working memory test requires retention and manipulation of visuospatial information. This self-ordered test has notable executive function demands and provides a measure of strategy as well as working memory errors. This test can therefore be used to demonstrate that there is a relationship between the reduction in walking speed from the TUG and the D-TUG and cognition in older people with mild cognitive impairment or early dementia.

\section{HOME FIRST - INTRODUCING A FRAILTY INTERVENTION AND RESPONSE TEAM TO AN ACUTE HOSPITAL EMERGENCY DEPARTMENT}

Dillon A., Edge L., O’Shaughnessy Í., Flynn S., Cunningham C. and Shields D.

St James's Hospital, James's Street, Dublin 8, Ireland

Introduction: A recent report by The Irish Longitudinal Study on Ageing (TILDA) recommended implementation of admission avoidance services and dedicated tailored care of the oldest old in emergency settings ${ }^{1}$. A Frailty Intervention and Response Team consisting of a Clinical Nurse Manager 2, Clinical Specialist Physiotherapist, Clinical Specialist Occupational Therapist and Senior Medical Social Worker was introduced to the Emergency Department (ED) of a Large Acute Teaching Hospital. Findings related to patient demographics \& discharge outcomes are reported below.

Methods: Quality Improvement Methodology underpinned the development of care pathways. Tests of change were performed using Plan Do Study Act (PDSA) cycles. Home FIRsT aimed to screen all patients $\geq 70$ years of age for frailty presenting to the Emergency Department within core working hours Monday to Friday. Microsoft Excel was used for data collection and analysis.

Results: In the first two months, 494 ED attendances were included, 189 male ( $38 \%$ ) and 305 female (62\%), mean age 78 (range 66-101). Twenty-six percent $(n=130)$ were living alone. Patients screened for frailty were Manchester triage category $2(7 \%, n=35)$, triage category $3(73 \%, n=359)$ and triage category $4(20 \%, n=98)$. Medical assessment was performed first in the case of Triage category 2. Sixty percent $(n=294)$ were discharged home from the ED. Fifteen percent of those $(n=72)$ were discharged with onward referral to Medicine for the Elderly Ambulatory care services i.e. Day Hospital, Bone Health Service, Falls \& Syncope Unit, Medicine for the Elderly OPD.

Conclusion: The introduction of a Frailty Intervention and Response Team enabled the commencement of Comprehensive Geriatric Assessment in the ED and promoted timely access to Medicine for the Elderly Ambulatory Care Services for those identified with markers of Frailty.
Reference

1. McGarrigle C, Donoghue O, Scarlett S \& Kenny RA (2017) 'Health and Wellbeing: Active Ageing for Older Adults in Ireland' Evidence from the Irish Longitudinal Study on Ageing http://tilda.tcd.ie/ assets/pdf/TILDA\%2OWave\%203\%20Key\%2OFindings\%20 report.pdf

\section{MULTI-AGENCY MULTI-DISCIPLINARY ENGAGEMENT IN DEVELOPING AN INTEGRATED EVIDENCE-BASED PATHWAY FOR FALLS PREVENTION AND CARE FOLLOWING A FALL}

Fleming J. ${ }^{1}$, Jalal H. ${ }^{2}$, Eastment $\mathrm{J}^{3}$, Tunster H. ${ }^{3}$, Riglin $\mathrm{J}^{2}$ and Mavrodaris A. ${ }^{1,3}$

'Cambridge Institute of Public Health, University of Cambridge; ${ }^{2}$ Cambridgeshire and Peterborough NHS Foundation Trust; ${ }^{3}$ Cambridgeshire County Council Public Health Directorate

Introduction: An integrated evidence-based pathway to reduce and manage falls amongst older people has been developed following NICE guidance by a multi-agency falls prevention working group lead by the Public Health ageing team in a UK county council (Cambridgeshire). To refine the pathway a pilot project tested implementation in one Neighbourhood Team area serving the population of 12 general practices. Before expanding roll-out county-wide, a falls prevention pathway workshop was held to map interdependencies, links and gaps to be addressed.

Methodology: Workshop invitations went to front-line staff and volunteers in multiple agencies operating in the pilot project area. Attendees were asked to focus small group discussion on structured questions and to provide written contributions detailing their current roles, where these fitted in the falls pathway, specific actions needed to effectively implement the pathway, perceived challenges and suggested solutions to help get system-wide falls prevention into practice.

Results: The workshop attracted over 60 attendees representing over 50 different services, teams, practices and agencies including health, social care, housing, emergency services and voluntary sector. Despite keen interest, lack of awareness of each other's falls-related services was striking and confidence in risk-reduction interventions' timeliness was low. Communication factors emerged as key themes: 1) Communication to raise awareness of preventable falls risk factors, referral routes, up-to-date information on local existing services, easy single-point-of-access especially for acute services serving multiple areas; 2) Individual-level communication between sectors, particularly the need for IT access to consistent assessment tools, information sharing with consent to avoid duplication and patient burden, referral tracking; 3) Empowering individuals and families through communication about what they can do, especially motivational support for exercise; 4) Voluntary sector potential communication and support roles.

Conclusions: Falls prevention is widely recognised as a 
priority by multiple stakeholders keen to engage. Optimum implementation requires time for communications development.

\section{USING VERTICAL AQUATIC EXERCISE FOR AGILITY TRAINING \\ Galloway M. \\ Fitness \& Lifestyles Department Jewish Community Centre of Greater Vancouver; Canada}

Introduction: Gravity may be best for promoting bone health but orthopaedic challenges can make exercise in gravity difficult. Swimming may offer cardiovascular conditioning but requires specific skills. Vertical water exercise allows safe movement for a wide range of fitness levels and age groups. The physiological benefits of vertical water exercise have been researched. Training aimed specifically to fall reduction is lacking. Lateral direction change, power (velocity \& force), reactions, arm/hand reaching speed, dynamic balance and peripheral visual quickness are aspects of agility that vertical aquatic exercise can offer. Heart health and social wellness add other important benefits. Falls during class may cause wet hair; not fractured hips.

Methods: To quote from European Review of Aging and Physical Activity 2012 9:97, "There is a lack of metaanalysis and systematic reviews to describe the effects of water-based exercise on physical fitness parameters in elderly subjects".

Over 20 years I have taught vertical aquatic exercise introducing "fall reduction" techniques as I have learned them. Water's resistance and support make it ideal for actions which many older people fear doing on land; lateral direction change being an excellent example. Research could lead to evidence based training for aquatic instructors who teach older adults at risk of falls.

Results: In the past decade there have been studies pointing to the benefits of aquatic exercise for cardiovascular and strength improvement in the older population, particularly those with orthopaedic concerns. There are no solid studies relating directly to fall reduction principles.

Conclusions: Research is needed to quantify the transferrable benefits which might be obtained from regular vertical water exercise towards reducing fall risks. There is also a need for instructor education addressing appropriate techniques to incorporate into classes at all levels of ability.

\section{PREDICTION OF FALLS IN PARKINSON'S DISEASE USING THE TIMED UP AND GO TEST AND BODY- WORN SENSORS}

Greene B.R. ${ }^{1}$, Caulfield B. ${ }^{2}$, Lamichhane D. ${ }^{3}$, Bond W. ${ }^{4,5}$, Svendsen J. ${ }^{4}$, Zurski C. ${ }^{4}$ and Pratt D. ${ }^{3,6}$

'Kinesis Health Technologies, Dublin DO4 V2N9, Ireland; ${ }^{2}$ nsight centre and Insight Centre for Data Analytics, University College
Dublin, Dublin 4, Ireland; ${ }^{3}$ OSF Health Care, Illinois Neurological Institute, Peoria, IL 61605, USA; ${ }^{4}$ Jump Simulation, OSF HealthCare, Peoria, IL 61605, USA; ${ }^{5}$ Dept. of Emergency Medicine, University of Illinois College of Medicine at Peoria, Peoria, IL 61605, USA; 6Saint Thomas Rutherford hospitalist services, Murfreesboro, TN 37129, USA

Introduction: Parkinson's disease (PD) is a progressive neurodegenerative disease affecting approximately 3 people in every 1000, this prevalence rises steeply with age. The costs associated with PD are very significant and, in the US alone have been estimated to be $\$ 23 B n$ per year.

PD patients are at much higher risk of falls than the general population and have a higher prevalence of falls in comparison to other neurological disorders. Current assessment methods for falls in PD are subjective and may be limited in accuracy. Objective assessment of PD patients using inertial sensors may be useful in assessing falls risk and monitoring disease progression.

Methodology: We report a single site longitudinal study of 15 PD patients, followed up for falls over a 6 month period, using weekly fall diaries. Participants were assessed, on a monthly basis, using a quantitative version of the Timed Up and Go (QTUG) test. We aimed to examine the predictive validity for falls of the QTUG falls risk estimate (FRE) and frailty estimate (FE).

Results: Weekly falls diaries were available for 15 participants. Complete follow-up data at 180 days were available for 12 of 15 participants, while complete followup data at 90 days were available for all 15 participants. At 90 days, 4 of 15 participants had experienced a fall, while at 180 days, 8 of the 12 participants remaining had experienced a fall. A total of 181 falls were recorded. The baseline QTUG FRE was 73.33\% [44.90, 92.21] accurate in predicting falls within 90 days, while the baseline frailty estimate and TUG time were $60.00 \%[32.29,83.67]$ and $46.67 \%[21.27,73.41]$ accurate respectively.

Conclusion: Results suggest that a QTUG assessment is more accurate than the TUG time alone in predicting falls in PD patients within 90 days of assessment.

\section{PREDICTION OF FALLS IN COMMUNITY DWELLING OLDER ADULTS USING BODY-WORN SENSORS} AND CLINICAL RISK FACTORS

\section{Greene B.R. ${ }^{1}$ and Caulfield B. ${ }^{2}$}

${ }^{1}$ Kinesis Health Technologies, Dublin, Ireland; ${ }^{2} B$. Caulfield, Insight centre and School of Physiotherapy and Performance Science, University College Dublin, Ireland

Introduction: Falls are the leading global cause of accidental death and disability in older adults, with an estimated one in three adults over 65 falling each year. Falls are also the most common cause of injury and hospitalisation in this age group, and in the UK alone are estimated to cost the NHS £4.6M per day. Accurate, early identification of patients at risk of falling 
could lead to timely and effective intervention, reducing the incidence of fall-related injury and reducing associated costs. Methods: We report an evaluation of a method for fall risk assessment in community dwelling older adults. This technology, called the Quantitative Timed Up and Go (QTUG ${ }^{\text {TM}}$ ) is a registered medical device that combines sensor-based and clinical data to provide an objective assessment of falls risk and frailty, as well as automatic comparison of patient data against a reference population. The sensor data is obtained using inertial sensors worn during the Timed Up and Go (TUG) test and the clinical data is obtained using standard clinical fall risk questionnaires. Results are compared against the results for the standard TUG test (timed with a stopwatch) as well as standard clinical risk factors on the same cohort.

Results: Results obtained using supervised classifier models validated through nested cross-validation, on a sample of 400 community dwelling older adults (see Table 1), show that a model based on a combination of clinical and sensorbased data approach yields a classification accuracy of $70.5 \%$, compared to $70.5 \%$ for sensor-based assessment alone, $59.5 \%$ for clinical risk factors or $61.9 \%$ for the TUG test taken alone.

\begin{tabular}{|l|c|c|c|c|}
\hline N=400 & Combined & QTUG & $\begin{array}{c}\text { Clinical risk } \\
\text { factors }\end{array}$ & TUG \\
\hline Acc (\%) & 70.50 & 70.50 & 59.50 & 61.9 \\
\hline Sens (\%) & 63.10 & 70.83 & 36.48 & 23.5 \\
\hline Spec (\%) & 75.86 & 70.26 & 79.30 & 89.5 \\
\hline PPV (\%) & 65.43 & 63.30 & 55.24 & 61.8 \\
\hline NPV (\%) & 73.95 & 76.89 & 64.06 & 61.9 \\
\hline
\end{tabular}

Table 1. Falls risk assessment results for combined sensor and clinical risk factors, sensor data alone, clinical risk factors alone and TUG test taken alone.

Conclusion: These results suggest that the combination of sensor and clinical fall risk data provides a more robust and accurate method for community based assessment of falls risk, when compared to clinical questionnaire data or the standard TUG test. Community implementation of objective fall risk assessment methods has the potential to prevent falls through early intervention, reduce costs, and improve the quality of care for older adults deemed at risk of falling.

\section{BARRIERS AND FACILITATORS TO ADHERENCE IN AN EXERCISE AND ACTIVITY PROGRAMME AMONG OLDER ADULTS WITH MILD COGNITIVE IMPAIRMENT AND EARLY DEMENTIA}

Hancox J., van der Wardt V., Pollock K., Vedhara K. and Harwood R.H.

Division of Rehabilitation and Ageing, University of Nottingham School of Medicine, Queen's Medical Centre, Nottingham, NG7 2UH. UK
Introduction: Older adults with dementia are at a high risk of falls, with $60-80 \%$ of individuals falling within one year of diagnosis. Promoting Activity, Independence and Stability in Early Dementia (PrAISED) is a 12-month person-centred exercise and activity programme which aims to increase activity and independence whilst reducing falls in people with early dementia. In this patient group, as well as many others, poor adherence to physical activity interventions can undermine treatment effectiveness. The aim of the present investigation is to explore the barriers and facilitators influencing participants' adherence to the PrAISED exercises and activities.

Methodology: Semi-structured face-to-face interviews were conducted with 20 individuals with mild cognitive impairment or early dementia and their carer(s). Qualitative data were transcribed verbatim and analysed thematically.

Results: The findings revealed the main facilitators reported by participants and their carers to be: enjoyment, experiencing health improvements, believing in the longterm benefits of the exercises, having previous experience of sport/exercise, feeling confident doing the exercises, getting into a routine, and receiving social support. The main perceived barriers to adherence included: lack of enjoyment, low energy, not believing that the exercises will improve their health, time, and feeling that they already do enough activity. Conclusion(s): The findings advance understanding of the barriers and facilitators to exercise engagement in older adults with early dementia and can be used to inform the development of future falls interventions with this target population.

\section{USING SIMPLE CLINICAL TOOLS TO IMPROVE NUTRITION AND EFFECTIVENESS OF PATIENT CARE}

Harkness M., Hanna P. and Nusco C.

York Teaching Hospital NHS Foundation Trust

Introduction: Nutrition is a key element of care for hip fracture patients as we aim to complete prompt surgery, early mobilization and continue daily post-operative therapy. Following a hip fracture, malnutrition is associated with impaired post-operative recovery and poorer overall outcomes. Poorly nourished patients display reduced muscular strength which may result in impaired mobility and delayed rehabilitation.

Methodology: Improvement project, involving the whole ward team to improve nutrition in hip fracture patients. Over the past two years, we have maintained a clear focus on nutrition using board rounds, ward rounds and particularly the weekly MDT which is attended by a Dietitian.

Results: Through a series of PDSA cycles, we established a new process. Physiotherapy colleagues now aim to weigh patients when they are first mobilized the day after surgery. Patients are then highlighted to be weighed at weekly 
intervals. At the MDT, the Dietitian reviews all food charts and these are discontinued in patients that are eating well and have a low nutritional risk score. All patients with hip fracture are offered a high protein supplement unless contraindicated (e.g. chronic kidney disease), where a suitable alternative is chosen. Based on nutritional risk screening nursing staff are given guidance on ways to attempt to improve a patient's dietary intake and when to refer to the Dietitian. The vast majority of referrals are now being made by telephone rather than through the weekly MDT. These improvements have enabled the MDT to focus more clearly on the highest risk patients and have highlighted those that have continued to lose weight despite optimized nutrition suggesting other potential underlying diagnoses.

Conclusion: Accurate measurement of nutritional risk assessment, weight and food intake are simple clinical tools that can be used effectively to assist the MDT to improve patient care and outcomes.

\section{EXPERIENCES OF FALLS IN DOMESTIC SETTINGS AND USE OF AMBULANCE SERVICES: AN ETHNOGRAPHIC STUDY OF NON-CONVEYED PATIENTS}

\section{Heaton C.}

Bridgewater Community Healthcare, Wigan. UK

Introduction: Each year, 35\% of people over age 65 experience falls and approximately $45 \%$ of those are aged over 85 and live in the community. Local unpublished data from the ambulance service found that of the call outs, $24-32 \%$ of the patients were non-conveyed, of this only $32-52 \%$ of these patients were referred to the local Falls Prevention Service for a specialist multifactorial assessment (NICE 2013).

Background: The review supported the need for falls pathways to be in place, but did not uncover the reasons for low referral rates of non-conveyed patients to falls prevention services. This presentation will outline the design of a study which aims to: a) gain an in-depth understanding of the patient journey from patients, carers, and ambulance crews' perspectives, $b$ ) generate a clear understanding of the ambulance service sub-cultures which could inform the need for improvement of the existing falls pathway or provide confirmation of an effective pathway.

Methods: An ethnographic approach enables interpretation within a culture and looks at themes, patterns of value, behaviours and beliefs that are explored. The target sample will be people over 50 years of age who have fallen and have been seen by the ambulance service, but have not been referred into the falls prevention service. I will also recruit ambulance crew.

Results: The goal of this study is to gain a deep and rich description of culture and subcultures. The findings will be presented to stakeholders, at conferences and in publications.

\section{AN EXEMPLAR COMMUNITY FOUNDATION NHS TRUST INTEGRATED FALLS MANAGEMENT \& FRACTURE LIAISON SERVICE}

Heaton C. Bridgewater Community Healthcare, Wigan, UK

Introduction: The Nurse Consultant lead service is an integrated multidisciplinary service that provides specialist assessment, diagnosis and treatment for patients who suffer with falls, reduced balance and fractures. It provides lifestyle advice, investigations into bone density and underlying conditions and promotes bone health and effective treatment for Osteoporosis. We provide therapeutic interventions to improve strength, balance, mobility and function, which aim to improve confidence, and reduce risk of falls and fractures and promotes independence.

Methodology: We assess patients who have had a fall or are at risk of falling and assess patient over 50 years old following a fracture. Through integrated working we provide effective and timely assessment and interventions for our patients. We work in partnership with the local acute NHS trust and other agencies such as local leisure trust, ambulance and fire services, local council, charities and national organisation such as the National Osteoporosis Society and their local patient forum. The established patient pathway ensures that patients who require secondary care are referred appropriately and in a timely manner, therefore providing a cost effective service.

Results:

- $95 \%$ of patients referred are seen under 6 weeks.

- $100 \%$ of patients assessed against NICE guidance.

- $100 \%$ of patients followed up after bisphosphates started.

- DNA rates FLS- 10\% / Falls- $4 \%$.

- Referral to secondary care- Falls- 8\%, Complex Osteoporsis- $6 \%$, other secondary care consultants 3\%.

- Dexa outcomes - $57 \%$ of patients assessed required a dexa scan, of theses; $41 \%$ had reduced bone density needing a follow up scan in 2-3 years; $34 \%$ required lifestyle advice only and $21 \%$ were diagnosed with Osteoporosis and medication was requested to their GP's.

Conclusions: An effective service providing timely and cost effective assessment, interventions and treatment.

\section{COMPARING MALNUTRITION SCREENING TOOLS IN A SECONDARY CARE FALLS CLINIC}

\section{Hoerlyck C. ${ }^{1}$, Baggott J. ${ }^{2}$, Wilkins A. ${ }^{2}$, Hood V. ${ }^{2}$ and Masud T. ${ }^{2}$ \\ 'Aarhus University, Denmark; ${ }^{2}$ Rehabilitation Unit Nottingham University Hospitals NHS Trust Nottingham, UK}

Introduction: Falls can lead to significant morbidity, hospitalisation, institutionalisation and societal cost. Sarcopenia is an emerging concept associated with falls. Poor nutrition is a risk factor for sarcopenia and studies suggest 
nutritional supplements are a useful intervention for the condition. This study compared three different malnutrition screening tools in the falls clinic.

Methods: Consecutive attenders at a falls clinic were recruited. Malnutrition risk was measured by three tools: Malnutrition Screening Tool (MST), Mini Nutritional Assessment-Short Form (MNA-SF) and Malnutrition Universal Screening Tool (MUST). Sarcopenia was diagnosed using the European Working Group on Sarcopenia in Older people definition and cut-offs. Skeletal muscle mass (MM) was measured by bio-impedance (Tanita BC-418), hand grip strength (GS) by a dynamometer, and the 10-m walking speed (WS) was ascertained.

Results: Fifty patients [mean age $=80.3$ years $; 26$ women] were recruited. Mean WS was $0.63 \mathrm{~m} / \mathrm{s}(\mathrm{SD}=0.22)$. Mean GS for men and women were $29.0 \mathrm{~kg}(\mathrm{SD}=8.8)$ and 17.5 $\mathrm{kg}(\mathrm{SD}=4.8)$; and mean $\mathrm{MM}$ were $7.9 \mathrm{~kg} / \mathrm{m} 2$ and $6.8 \mathrm{~kg} /$ $\mathrm{m} 2$ respectively. Overall $61.4 \%$ (27/44) were sarcopenic. Patients screened positive for malnutrition risk were $2.4 \%$ (1/41), 34.0\% (17/50) and 60.0\% (30/50) with MUST, MST and MNA-SF respectively. MUST only screened $4.3 \%$ of the sarcopenic patients as at risk of malnutrition, whereas MST screened $44.4 \%$ and MNA-SF screened $66.7 \%$. No patients screened positive to all three tools. Of 24 patients screened positive by MNA-SF, 12 were also screened positive by MST, whereas of 13 patients screened positive by MST, 12 were screened positive by MNA-SF.

Discussion: There was significant mismatch between the three tools used in this population with a high sarcopenia prevalence. MUST underperforms substantially in screening for malnutrition in comparison to MST and MNA-SF. Further work is required to assess which tool performs with the best accuracy when compared to more comprehensive gold standard nutrition assessments in fallers.

\section{DO BEDRAILS REDUCE THE INCIDENCE OF FALLS IN ELDERLY NURSING HOME RESIDENTS COMPARED TO NO BEDRAILS}

\section{Huynh D., Lee O., Mannion C., Ens T. and An M.}

University of Calgary, Faculty of Nursing, Canada

Introduction: During clinical placements, we noticed controversy regarding the use of bedrails for fall prevention in elderly clients ( $>65 \mathrm{y}$ ). Some healthcare providers believed bedrails prevent falls, while others believed bedrails could result in worse injuries.

Methodology: We searched HealthStar, CINAHL, MEDLINE, and Academic Search complete to address the question: "Does the use of bedrails for older adults in Long Term Care reduce the incidence of falls compared to not using bedrails?" Proquest and Canadian Health Research Collection were searched for relevant grey literature. Two hundred and five records were identified. 86 remained after duplicates were removed. Abstracts and titles were scanned for relevance.
Grey literature were scanned for the text "bedrail"or "siderail" and relevance. Records that were not original or focused on a different population were excluded. Those that addressed falls and stated either "bedrail", "siderail", or "restraint" were included. Twenty-seven full text articles were screened. One non-english record was excluded. Many articles did not specify the age range of their sample. However, they were included as the average age was $>65$ or the participants resided in nursing homes.

Results: Fourteen articles remained and were used in our systematic review. The results suggest that bedrails do not affect fall frequency and may lead to more severe injuries when used. When interventions focused on bedrail reduction and fall prevention are used, fall frequencies were not affected. However, it is uncertain if fall frequencies will remain the same if staff do not implement other fall prevention strategies in addition to bedrail reduction.

Conclusion: Bedrails do not reduce the frequency of falls and may result in more severe fall related injuries.

\section{CLINICAL FRAILTY IN PATIENTS WITH HIP FRACTURES}

\author{
Karpinski S. ${ }^{1}$, Coary R. ${ }^{1}$, Farrelly A. ${ }^{1}$, Soh J., Hurson C. ${ }^{2}$ \\ and Doyle R. \\ 'Department Medicine for Elderly, St. Vincent's University Hospital, \\ Dublin Ireland; 'Department of Trauma and Orthopedics, St. \\ Vincent's University Hospital, Dublin Ireland
}

Background: Frailty is a clinical state of increased vulnerability resulting from age-associated physiologic decline ${ }^{1}$. Hip fractures are among the most serious injury due to fall. They result in loss of independence with $50 \%$ unable to walk independently again, difficulties in carrying out activities of daily living and around a third die within one year' ${ }^{2}$.

Methods: We screened patients admitted to Acute Orthopedic Unit with hip fractures from November 2015 until June 2016.These same patients were followed up in orthogeriatric virtual clinic a year later. The clinical frailty (CFS) was assessed using the Rockwood Frailty Index ${ }^{3}$. Other assessments included Zuckerman Hip Function Recovery Score and by New Mobility Scale (NMS).

Results: In total 109 patients were admitted with an average age of 84 . The average CFS was 5.5, average Zuckerman score was 63.1, and average NMS was 4.91. One year later, there were $73 \%$ survivors, average age 82 . CFS was 5.3 , average Zuckerman score was 55.1 and average NMS was 4.29. Five patients were lost to follow up. Mortality at one year was at $22 \%$. The average age of these patients on admission was 86 , average CFS was 6 , average Zuckerman score was 38 and average NMS was 2.5. One year later, $55 \%$ that were alive had a CFS equal or greater than 6 .

Conclusion: Hip fracture patients are frail (average clinical frailty score was 5 ). One year post hip fracture there was 
a deterioration in clinical frailty which corresponded with decrease in Zuckerman score (63 to 55) and in New Mobilty Scale (5.5 to 4.29).

\section{References}

1. Hubbard $R$, Theau O. Frailty: enhancing the known knows. Age Ageing (2012) 41(5): 574-575.

2. Neuburger $J$, Currie $C$, Walkeman $R$ et al. The impact of national clinic led audit initiative on care and mortality after hip fracture in England: external evaluation using time trends in non-audit data. Med Care 2015:53:686-91.

3. Rockwood K, et al. A global clinical measure of fitness and frailty in elderly people CMAJ (2005) vol: 173 no.5.

\section{FRACTURE LIASION SERVICES: FRAGILITY FRACTURE AND FALLS PREVENTION IN THE UK}

\section{Doyle A. and Carr W.}

National Osteoporosis Society, UK

Introduction: The National Osteoporosis Society aims to ensure that every person aged over 50 who breaks a bone is assessed for osteoporosis, managed appropriately through a Fracture Liaison Service (FLS) and referred to Falls Services where appropriate. FLS prevent secondary fractures using dedicated case-finding to identify fragility fractures, with assessment and treatment of osteoporosis where necessary. Close links between FLS and Falls Services ensure that patients at risk of falls are evaluated, and referred for appropriate interventions.

Methodology: A gap analysis tool was used to measure service provision against two of the Clinical Standards for FLS relating to evaluation of falls risk within prescribed timescales; and referral of patients at increased risk of falls for intervention to reduce future risk. Data was collected at 87 sites across the UK between 2015 and 2017.

Results: $23 \%$ of sites did not offer a falls risk assessment within three months of incident fracture.

$22 \%$ of sites had no system in place to refer patients identified as being at risk of falls, for intervention to reduce that risk.

Discussion: Timely intervention and treatment of fragility fractures is essential to prevent further fractures. Up to $11 \%$ of hip fracture patients will sustain another hip fracture, and $20 \%$ of female vertebral fracture patients will sustain another vertebral fracture in the first year. Patientcentred services must include falls risk assessment in their evaluation of future fracture risk, to optimise the patient pathway and reduce secondary fractures.

Conclusion: When services operate to national clinical standards and deliver collaborative care-planning, future risk of falls is reduced by up to $30 \%$. Gap analysis highlights the need of a significant minority of sites to review their current clinical pathway in respect of falls risk assessment and referral to fully implement the standards and address the challenge of falls-related fragility fractures.
THE IMPACT OF VERTEBRAL FRACTURES ON HEALTH RELATED QUALITY OF LIFE IN OSTEOPOROTIC PATIENTS ATTENDING A BONE HEATH SERVICE

Maher N., Fallon N., Steen G., Brown P., Mahon J., McCarroll K., Walsh J.B. and Casey M.

Bone Health and Osteoporosis Unit, Mercers Institute for Successful Ageing, Misa Building, St James's Hospital, Dublin, Ireland

Introduction: Vertebral fractures are a common complication of osteoporosis and are associated with back pain and functional impairment, which can influence mood and lead to depression. This can have a negative impact on patient health related quality of life (HRQOL).We investigated the effect of vertebral fractures on HRQOL in osteoporotic patients.

Methods: A cross sectional study was carried out amongst patients attending a bone health service for osteoporosis assessment. 88 patients with vertebral fractures and 85 patients with no vertebral fractures were included. All patients completed the Quality of Life Questionnaire of the European Foundation for Osteoporosis (QUALEFFO) on first attendance. Each patient recieved Dual Emission xray absorptimetry (DXA). Vertebral fractures were diagnosed by a lateral vertebral assessment (LVA). The QUALEFFO is a disease specific $\mathrm{HRQOL}$ questionnaire targeting fracture assessment specific to patients with vertebral fractures. It comprises 41 questions in 5 domains: Pain; Physical Function; Social Function; General Health Perception and Mental Function.

Results: 173 community dwelling osteoporotic patients attending a bone health and osteoprosis clinic were included. $7: 1$ female to male ratio. Mean age 77 years (Range 31-92). $64 \%$ patients had previous fractures with $66 \%$ taking bone health medication on presentation. $14 \%$ were treatment naive. Patients with vertebral fractures experienced significantly more moderate - severe pain $(p=0.0001)$ and reported significant reduction in ADLs $(p=0.003)$. They were significantly less mobile ( $p=0.0005$ ) were more socially isolated $(p=.003)$ and reported a significant reduction in perceived general health $(p=0.004)$ and perceived QoL ( $p=0.001)$.

Conclusions: Our results support previous findings that HRQOL is significantly decreased in patients with vertebral fractures. It highlights the importance of preventing, diagnosing and treating vertebral fractures to reduce their negative impact on HRQOL.

\section{PREVALENCE AND OVERLAP OF VASOVAGAL SYNDROME, CAROTID SINUS SYNDROME AND ORTHOSTATIC HYPOTENSION DIAGNOSED BY TILT TABLE TESTING}

Mansour S., Lwin Z., Banu S., Shahpar K., Poon M.H. and Masud T.

Nottingham University Hospitals NHS Trust, UK.

Introduction: Tilt testing is increasingly being recognised as important in the assessment of unexplained syncope, falls 
and dizziness. Vasovagal syndrome (VVS), carotid sinus syndrome (CSS) and orthostatic hypotension $(\mathrm{OH})$ are the commonest diagnoses resulting from tilt testing, although there is overlap of these diagnoses in many patients. The aim of this study was to determine firstly the prevalence of these conditions and the extent of their overlap in patients referred for tilt testing to our department.

Methodology: We reviewed retrospectively results of patients consecutively referred for tilt testing. Diagnostic criteria were: $\mathrm{OH}=$ systolic or diastolic blood pressure (BP) drop of $\geq 20 \mathrm{mmHg}$ and $\geq 10 \mathrm{mmHg}$ respectively within 3 minutes of upright tilting; VVS=presyncope or syncope with associated BP drop $\geq 50 \mathrm{mmHg}$ on tilting at 70 degrees up to 40 minutes with sublingual glyceryl trinitrate provocation after 20 minutes; $C S S=$ carotid sinus massage producing $\geq 3$ second asystole (cardioinhibitory type) or systolic BP drop of $\geq 50 \mathrm{mmHg}$ (vasodepressor type) or presence of both (mixed type).

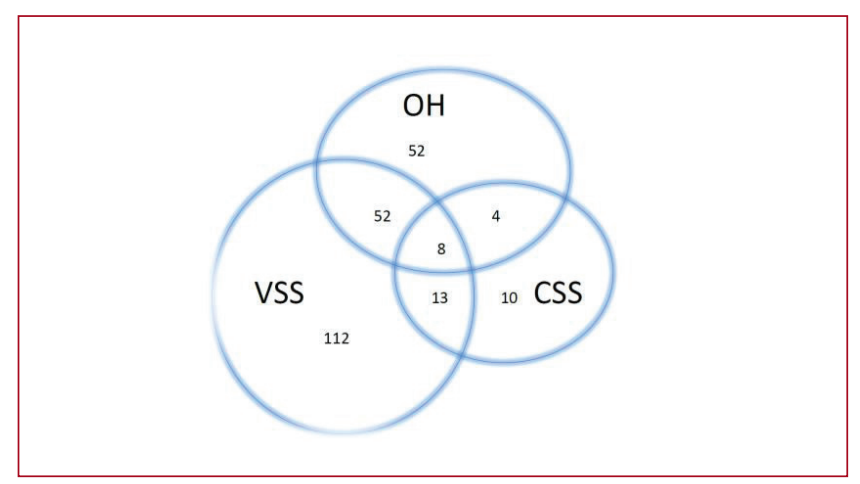

Results: 380 patients (235 female) mean age $=53.6$ years $(S D=22.5)$ were studied. Overall positive yield (any diagnosis) was $72.9 \%$. The prevalence of VVS, CSS and $\mathrm{OH}$ were $51.8 \%, 9.2 \%, 30.5 \%$ respectively. Overlap is shown in the Venn diagram. Of the 193 VVS patients, $31.0 \%$ also had $\mathrm{OH}$ and $10.9 \%$ had CSS. Of the $116 \mathrm{OH}$ patients $51.7 \%$ had VVS and $10.3 \%$ had CSS. Of the 35 CSS patients $34.2 \%$ had $\mathrm{OH}$ and $57.1 \%$ had VVS. Of the 277 patients with a positive tilt test $2.9 \%$ had all three diagnoses.

Conclusion: Positive yield of tilt testing is high. There is much overlap between VVS, CSS and $\mathrm{OH}$. In individual patients with unexplained falls, syncope and dizziness; tilt testing can therefore provide useful diagnostic information that potentially allows appropriate targeted treatment and advice.

\section{AN INVESTIGATION INTO THE PERCEPTION OF THE TERM "FRAILTY" AMONG MIDDLE-AGED AND OLDER ADULTS}

Lim J., Kenny L., Duggan O., McCullagh R. and Timmons S. Centre for Gerontology and Rehabilitation, School of Medicine, University College Cork, Ireland

Introduction: "Frailty" as a term is increasingly used by clinicians and researchers to define persons at risk of adverse outcomes. However, limited studies have examined what the term "Frailty" means to older persons. Existing definitions of the term may not encompass its unique meaning to the layman. Therefore, this study aims to build on existing conceptual understanding and add to the discussion towards standardizing the operational definition of the term "Frailty". Methods: This was a cross-sectional study. Communitydwelling participants, aged fifty and older, were recruited by purposive sampling, through social and retirement groups in Cork city and county, using additional snow-ball sampling. Participants were invited to complete a self-administered questionnaire.

Results: Fifty-eight questionnaires were distributed. Four were not returned ( $93 \%$ response rate) and three were incomplete. Of the final forty-one females and ten male respondents, $47.1 \%$ were aged 50-70. Twenty-nine were non-frail $(56.9 \%)$, fifteen pre-frail and seven frail. "Frailty" was largely perceived in terms of its physical dimension (64.7\%), followed by psycho-social components (14.1\%) and medical illness (13.5\%). A small proportion perceived "Frailty" to mean "old" $(7.6 \%)$. Almost half of the respondents believed that "Frailty" is preventable (45.1\%). These respondents gave examples of preventative action such as keeping active through regular exercise, maintaining a healthy diet, keeping a positive outlook to life and having a social support network. Those who felt that "Frailty" was not preventable believed it was fate, a natural result of ageing or attributed to bad genes.

Conclusion: The results provide a reflection of the meaning of the term "Frailty" to middle-aged and older persons. Those who perceived "Frailty" in physical terms, such as weakness and gait difficulties, may be open to physicallyfocused preventative strategies. However, work is needed to help engage individuals who do not perceive "Frailty" as preventable, thereby helping to shape successful ageing strategies.

\section{PHYSICAL PERFORMANCE AND QUALITY OF LIFE IS IMPROVED IN FRAIL OLDER MEDICAL INPATIENTS WITH AN AUGMENTED PRESCRIBED EXERCISE PROGRAMME}

\section{McCullagh R., O'Connell E., O'Meara S., Horgan N.F., Timmons S. \\ Centre for Gerontology \& Rehabilitation, College of Medicine, University College Cork, Ireland}

Introduction: Aim: To measure the effects of an augmented prescribed exercise programme on physical performance, quality of life and healthcare utilisation for frail older medical patients in the acute setting.

Methods: Within two days of admission, older medical inpatients with an anticipated length of stay $\geq 3$ days, needing assistance/aid to walk, were blindly randomly allocated 
to the intervention or control group. Until discharge, both groups received twice daily, Monday-to-Friday half-hour assisted exercises, assisted by a staff physiotherapist. The intervention group completed tailored strengthening and balance exercises; the control group, stretching and relaxation exercises. Physical performance (Short Physical Performance Battery), quality of life (QuroQOL-5D-5L), length of stay and readmission rates were measured at discharge and at three months. Time-to-event analysis was used to measure differences in length of stay. Changes in physical performance and quality of life was compared using unadjusted and adjusted linear regression models.

Results: Data from 198 patients (aged $80 \pm 7.5$ years) was analysed. Groups were comparable at baseline. Unadjusted analysis showed that the intervention reduced the length of stay slightly with no statistical significance (HR 1.09 $(\mathrm{Cl}, 0.77-1.56) \mathrm{p}=0.6)$. When patients transferred to rehabilitation were excluded and adjusted for confounders, the effect was greater, but remained insignificant $(n=125$, $1.3(\mathrm{Cl}, 0.9-1.87) \mathrm{p}=0.16)$. Adjusted and unadjusted physical performance was significantly and clinically meaningfully better in the intervention group at discharge (adjusted, $\mathrm{n}=174,0.78 \mathrm{Cl}, 0.28-1.29) \mathrm{p}=0.003$ ), but none at follow-up $(n=123,0.45(\mathrm{Cl}, 0.43-1.33) \mathrm{p}=0.3)$. When adjusted, quality of life was significantly and clinically meaningful better in the intervention group at discharge $(n=174,6.72(\mathrm{Cl}, 0.66-12.8) \mathrm{p}=0.03)$.

Conclusion: The significant changes in physical performance and quality of life suggest that this simple intervention is valuable and useful to frail medical inpatients.

\section{FALLS: THINK UNCONVENTIONALLY}

\section{McDonald L. and Tay H.S.}

Department of Geriatric Medicine, Aberdeen Royal Infirmary. NHS Grampian

Case History: A 79-year-old previously fully independent male presented initially to Geriatric Medicine clinic with poor balance, back and leg pain with stiffness progressing over five months. Whilst awaiting investigations he was admitted to hospital a month later following a fall down stairs, and with a history of rapid decline, recurrent falls and reduced activities of daily living due to reduced dexterity. There were no hallucinations or memory decline reported.

He was found to have increased tone on left side but no tremor or bradykinesia. Power, reflexes and sensation were normal but he had marked dyspraxia of his left upper limb, with no evidence of alien limb. Cranial nerve tests were normal. His Montreal Cognitive Assessment score was 8/30.

CT head showed small vessel disease and bilateral small established lacunar infarcts, with age related atrophy.

Initial clinical impression was possible Parkinson's Plus syndrome and a neurologist review was sought where he was diagnosed with Corticobasal Syndrome. Supportive management was provided.
Discussion: Corticobasal syndrome is a rare sporadic progressive neurological disorder with onset typically between 50 to 70 years old. It is thought to be a tauopathy'. Classic symptoms are asymmetric rigidity with apraxia and akinesia. Usually unilateral but progresses to bilateral and can affect all limbs. Cognitive symptoms are common including visual-spatial impairment, impairments in executive functioning and dementia 1 . It can progress over six to eight years and death usually occurs due to complications of immobility ${ }^{2}$. Treatment such as Levodopa has been tried with poor response and management is directed to symptom control $^{2}$. Multidisciplinary input such as physiotherapy, occupational therapy and speech and language therapy is beneficial ${ }^{3}$. Distinguishing corticobasal degeneration from other similar neurodegenerative disorders is difficult and with fairly rapid progression it is important to consider this as a diagnosis to aid in management of symptoms and anticipatory care planning ${ }^{4}$.

\section{References}

1. Progressive Supranuclear Palsy Association. A guide to PSP and CBD for General Practitioners and the Primary Healthcare Team. https://www.pspassociation.org.uk/app/uploads/2017/01/AGuide-to-PSP-CBD-for-GPS-and-the-Primary-Healthcare-Team-1. pdf. Accessed on May 11, 2017

2. National Institute of Neurological Disorders and Stroke. Corticobasal degeneration fact sheet. https://www.ninds.nih.gov/Disorders/AllDisorders/Corticobasal-Degeneration-Information-Page. Accessed on May 11, 2017

3. Lamb R, Rohrer JD, Lees AJ, Morris HR. Progressive Supranuclear Palsy and Corticobasal Degeneration: Pathophysiology and Treatment Options. Curr Treat Options Neurol. 2016;18(9):42.

4. Armstrong MJ, Litvan I, Lang AE, et al. Criteria for the diagnosis of corticobasal degeneration. Neurology. 2013; 80(5): 496-503.

THE ASSOCIATION BETWEEN BALANCE AND FREELIVING PHYSICAL ACTIVITY IN OLDER COMMUNITY DWELLING ADULTS

\author{
McMullan I., Bunting B., McDonough S., Tully M. and \\ Casson K. \\ Ulster University,Northern Ireland
}

Background: Poor balance is associated with an increased risk of falling, disability and death in older adults. Evidence suggests that exercise, a sub-category of physical activity (PA), that is planned and structured, is among the most important lifestyle factors for good balance, but less is understood about the effects of other types of PA domains. This novel study evaluates the relationship between balance and free-living physical activity (PA), the activity of everyday living, in older community-dwelling adults, to better inform future fall prevention programmes.

Methods: Measures of balance and free-living PA across a 2-year period were obtained from The Irish Longitudinal study of Ageing (TILDA) study. Data were analysed using a Structural Equation Modelling (SEM) approach to firstly 
identify an appropriate model of balance, and then to understand and explain the patterns of change in balance and PA over time controlling for other exogenous variables. Results: Free-living PA improves balance measured using Timed Up and Go (TUG), handgrip strength, Mini Mental State Exam, vision, hearing over a 2-year period. The exogeneous variables of sex, age, medication use, fear of falling, education, pain, alcohol consumption, and ADL disability were found to be significant risk factors for balance.

Conclusion: The findings in this study support that balance declines with age; that free-living PA improves balance in an older community-dwelling population; and that females, those in older age groups, medication, fear of falling, lower education attainment, pain, higher alcohol consumption, and the presence of ADL disability have an increased risk of poor balance.

\section{ORTHOSTATIC HYPOTENSION, ORTHOSTATIC INTOLERANCE AND FEAR OF FALLING}

\section{McNicholas T., O'Callaghan S. and Kenny R.A.}

The Irish Longitudinal Study on Ageing, Trinity College Dublin, Ireland

Introduction: Fear of falling (FOF) is defined as a lasting concern about falling leading to an individual avoiding activities that he/she remains capable of performing. It has been associated with depression, poor self-rated health, reduced social activity and impaired mobility. Orthostatic hypotension $(\mathrm{OH})$ is defined as a sustained reduction in systolic blood pressure (SBP) $\geq 20 \mathrm{mmHg}$ or diastolic blood pressure (DBP) $\geq 10 \mathrm{mmHg}$ within $3 \mathrm{~min}$ of standing. The aim of this study was to assess whether individuals with $\mathrm{OH}$ or orthostatic intolerance (OI) are more likely to report FOF.

Methods: Data from wave 3 of The Irish Longitudinal Study on Ageing (TILDA) were used. Beat-to-beat BP was measured during active stand lasting 110 seconds using a finometer. To assess for OI, participants were asked if they were dizzy on stand. Sustained $\mathrm{OH}$ was defined as a drop in SBP $>=20$ $\mathrm{mmHg}$ or a drop in DBP $>=10 \mathrm{mmHg}$ sustained at $40(\mathrm{OH} 40)$ and $110(\mathrm{OH} 110)$ seconds. As part of a computer aided personal interview (CAPI), participants were asked questions in relation to presence of FOF.

Results: There were 3574 participants who had active stand data that was adequate for analysis. Univariate logistic regression showed that individuals with $\mathrm{OH} 4 \mathrm{O}$ were more likely to report FOF (OR 1.57, $p=0.007$ ), however this association was not significant on controlling for potential confounders (OR 1.17, $\mathrm{p}=0.395$ ). There was no association between $\mathrm{OH} 110$ and FOF on univariate or multivariable analysis. OI was associated with FOF on univariate logistic regression (OR 1.64, $\mathrm{p}<0.001$ ) and this remained significant when controlling for confounders (OR 1.34, $p=0.046$ ).

Conclusion: There was no significant association between $\mathrm{OH}$ and FOF, however $\mathrm{Ol}$ is associated with an increased risk of FOF. Further analysis of the TILDA data using near infrared spectroscopy to measure cerebral perfusion on orthostasis may help to investigate this further.

\section{THE RELATIONSHIP BETWEEN GAIT SPEED, COGNITIVE IMPAIRMENT AND DUAL TASK CONDITIONS IN FALLERS AND NON-FALL CONTROLS}

\begin{abstract}
Minet L.R. ${ }^{1,2}$, Thomsen K. ${ }^{3}$, Ryg J. ${ }^{3}$ Matzen L. ${ }^{3}$, Ytterberg C. ${ }^{1,4,5}$, Masud T. ${ }^{3,6}$

'Research Unit of Rehabilitation, Department of Clinical Research, University of Southern Denmark, Denmark; '2Center for Applied Research, University College Lillebaelt, Denmark; ${ }^{3}$ Department of Geriatric Medicine, Odense University Hospital, Denmark; ${ }^{4}$ Department of Neurobiology, Care Sciences and Society, Karolinska Institutet, Sweden; ${ }^{5}$ Functional Area Occupational Therapy and Physiotherapy, Karolinska University Hospital, Sweden; ${ }^{6}$ Nottingham University Hospitals NHS Trust, United Kingdom
\end{abstract}

Introduction: Abnormal gait is an established falls risk factor and gait speed (GS) of $<0.6 \mathrm{~m} / \mathrm{s}$ is associated with falls. Cognitive impairment (Cl) and dual task conditions (DT) have been linked to impaired gait. We aimed to explore the relationship between GS, Cl and GS-DT in women with and without a falls history.

Methods: This case-control study was carried out in a group of fallers aged $\geq 65$ years recruited from a falls clinic [FALLCLIN] ( $n=114)$ and from age matched faller (FALLCON] $(n=97)$ and non-faller [NOFALIL] $(n=90)$ community controls. GS was evaluated using a 4-meter walking test with and without a DT condition at participant's preferred speed and with their customary walking aids. $\mathrm{Cl}$ was screened by the Mini Mental State Examination (MMSE).

Results: The overall median age (range) was 80 years (7586) and $74 / 301$ (24.6\%) had Cl. In the cognitively normal women (MMSE $\geq 25$ ) the median (IQR) GS (m/s over $4 \mathrm{~m}$ ) in the FALLCLIN, FALLCON and NOFALL groups were 0.86 (0.63-1.06), 1.11 (0.87-1.33) and 1.30 (1.06-1.58) [ $\mathrm{p} \leq 0.001$ ], and under DT conditions (counting backwards) were $0.67(0.46-0.80), 0.79(0.63-0.98)$ and $1.04(0.82-$ $1.28)[p \leq 0.001]$ respectively. In the $\mathrm{Cl}$ women (MMSE<25) the median GS in the FALLCLIN, FALLCON and NOFALL groups were 0.66 (0.56-0.79), 0.68 (0.62-1.04), 1.12 (0.85-1.35) [ $\mathrm{p} \leq 0.001]$; and under DT conditions were 0.46 (0.38-0.65), 0.49 (0.36-0.79) and $0.72(0.53-0.89)$ $[p=0.006]$ respectively.

Conclusion: GS was slower in the faller groups, in those with $\mathrm{Cl}$ and when DT conditions were present. In the presence of both $\mathrm{Cl}$ and DT conditions, the GS in the faller groups were very low and in the range known to be associated with increased falls risk. GS in normal and under DT conditions may be useful biomarkers for falls risk in cognitively impaired older people and further research is needed to explore if DT training can improve GS. 


\section{CIRCULATING IL- 18 IS HIGHER IN OLDER ADULTS WITH FRAILTY: THE IRISH LONGITUDINAL STUDY ON AGEING (TILDA)}

O'Halloran A. ${ }^{1,3}$, Connelly E. ${ }^{2}$, Kenny R.A. ${ }^{1,3}$ and Doyle S. ${ }^{2}$ 'Medical Gerontology, Trinity College, Dublin, Ireland; ${ }^{2}$ Clinical

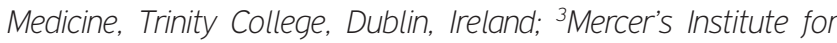
Successful Ageing, St James's Hospital, Dublin, Ireland

Background: Previously, a genetic variant in the gene for the pro-inflammatory cytokine interleukin-18 (IL-18) was associated with the Frailty Index (FI) in a large study of older adults (Mekli et al, 2015). Here we investigated the relationship between frailty and circulating IL-18 in older adults.

Methodology: Cross-sectional analyses were performed in a subset of adults [ $n=547$; Mean age: 65 years; $55 \%$ female] from the Irish Longitudinal Study on Ageing (TILDA). Total IL-18, IL-18 binding protein (IL-18BP) and free IL-18 were measured by enzyme-linked immunosorbent assay (ELISA) from frozen plasma samples. Pre-frailty (FI: 0.10-0.24) and frailty ( $\mathrm{Fl}: \geq 0.25$ ) were measured using a 32-item FI. Multinomial logistic regressions computed associations between IL- 18 levels and frailty status. Models were adjusted for age, age ${ }^{2}$, sex, education, C-reactive protein (CRP), BMI, smoking and physical activity.

Results: The proportion of the sample with pre-frailty and frailty was $33.3 \%$ (Mean Fl: 0.16) and $11.7 \%$ (Mean Fl: 0.34 ) respectively. Total and free IL-18 levels were higher among the pre-frail versus non-frail (28.5 vs. $23.7 \mathrm{pg} / \mathrm{ml}$; $\mathrm{p}=0.03$ ) and (25.6 vs. $21.2 \mathrm{pg} / \mathrm{ml} ; \mathrm{p}=0.03)$, but not among the frail. CRP levels were significantly higher among the prefrail (3.8 ng/ml; $p<0.01)$ and the frail $(4.7 \mathrm{ng} / \mathrm{ml} ; \mathrm{p}<0.01)$ versus the non-frail $(2.7 \mathrm{ng} / \mathrm{ml})$. In the fully adjusted model, an increase of one standard deviation of total IL-18 was significantly associated with both pre-frailty (RRR: 1.27; $p=0.02$ ) and frailty (RRR: 1.42; $p=0.02$ ). A similar pattern was seen for free IL-18 for both pre-frailty (RRR: 1.26; $p=0.02$ ) and frailty (RRR: $1.41 ; p=0.02)$. There was no association with IL-18BP.

Conclusions: This is the first study showing that total and free IL-18 were significantly associated with pre-frailty and frailty after adjustment for other markers of chronic inflammation and health. Circulating IL-18 may be a more informative marker of inflammaging and frailty than distal acute phase markers such as CRP.

\section{DIET QUANTITY NOT QUALITY, AGE, BMI AND SPORTING ACTIVITIES ARE PROLIFIC PREDICTORS OF BONE HEALTH IN MIDDLE-AGED AND OLDER PERSONS}

\author{
Tomlinson D.J., Morse C.I. and Onambele-Pearson G.L. \\ Manchester Metropolitan University, UK; Liverpool John Moores \\ University, Liverpool, UK
}

Introduction: The impact of independent modulators of bone health, including ageing, adiposity/obesity, nutrition and physical behaviour, is unclear. For instance, it would be expected that diets high in saturated fats negatively impact on the absorption of dietary calcium and ultimately bone health ${ }^{3}$. Similarly, high adiposity is associated with elevated systemic inflammation ${ }^{2}$ and hence does not favour bone health.

Methods: Using 50 untrained individuals aged 43-80, we contrasted the modulation of bone mineral density (BMD) through adiposity, habitual nutrition and physical behaviour. We hypothesised that: (i) optimal dietary composition (low saturated fats, high Calcium, Vitamin D, Vitamin C, Protein, Omega 3 and 6 Fatty Acids, Vitamin K, Zinc, Magnesium and Phosphorus) would promote bone health; (ii) the negative impact of high adiposity would be greater on under-loaded bone sites ${ }^{1}$; (iii) high physical activity more so than low sedentary behaviour would improve BMD ${ }^{4}$.

Results: All participants habitually consumed low saturated fat ( $<11 \%$ daily total diet). Overall diet quality did not differ between subpopulations except for total protein intake difference between low and high adipose persons ( $p=0.049)$. Similarly, habitual physical behaviour was not a determinant of BMI, except for a trend towards leisure time activity difference between normal weight and obese individuals $(p=0.054)$. Diet quality also did not predict BMD in any of the bone sites. Interestingly, sporting activities predicted 4/5, age, BMI and total calories each predicted $3 / 5$, global physical activity and adiposity each predicted 1/5, BMD sites of interest (Table 1 ). Only one BMD site differed between low vs. high adipose persons $(p=0.025)$. BMI however differentiated normal weight and obese individuals in 2/5 BMD sites ( $p \leq 0.024)$.

Conclusions: BMD 'optimization focused lifestyle interventions' should target education regarding adequate food quantity intake, whilst aiming also to increase habitual moderate-to-vigorous physical activity, particularly in middle-aged to older individuals.

Table 1. Pearson product moment and Spearman rank order correlations ( $r$ ) between age, physical activity scores, adiposity, $\mathrm{BMI}$, and habitual nutritional intake against a series of bone sites BMD i.e. Bone Mineral Density; $\left({ }^{*} p<0.05\right.$, ${ }^{* *} p<0.01$, $* * * 00.001)$.

\begin{tabular}{|c|c|c|c|c|c|c|c|c|c|c|}
\hline \multirow[b]{2}{*}{ Bone } & \multirow[b]{2}{*}{ (1) Age } & \multicolumn{4}{|c|}{ Physical Activity Score (2) } & \multirow[b]{2}{*}{ Adiposity (3) } & \multirow[b]{2}{*}{ BMI (4) } & \multicolumn{3}{|c|}{ Nutrition (5) } \\
\hline & & Work (i) & Sport (ii) & Leisure(iii & i)Global (iv) & & & Total score (i) & Bone score (ii) & Total Calories (iii) \\
\hline Total BMD & $-.423 * *$ & .095 & $.349 *$ & -.122 & .208 & .061 & .260 & -.194 & -.206 & $.404^{* *}$ \\
\hline Lumbar BMD & -.238 & -.124 & $.312 *$ & -.168 & .012 & -.063 & .041 & -.045 & -.009 & .151 \\
\hline Pelvis BMD & $-.450 * *$ & .091 & $.399 * *$ & .100 & $.355^{*}$ & .173 & $.284^{*}$ & -.192 & -.210 & .194 \\
\hline Arm BMD & -.194 & .113 & .262 & -.061 & .170 & $.307^{*}$ & $.496^{* * *}$ & -.221 & -.178 & $.471^{* *}$ \\
\hline Leg BMD & $-.281^{*}$ & .138 & $.348^{*}$ & -.090 & .252 & .184 & $.385^{* *}$ & -.160 & -.124 & $.475^{* * *}$ \\
\hline
\end{tabular}




\section{References}

1. Devlin M and Rosen CJ, 2015. The Bone Fat Interface: Basic and Clinical Implications of Marrow Adiposity. Lancet Diabetes Endocrinol 2015 February; 3(2): 141-147. doi:10.1016/S22138587(14)70007-5.

2. Erskine RM, Tomlinson DJ, Morse Cl, Winwood K, Hampson P, Lord JM, and Onambele GL, 2017. The individual and combined effects of obesity- and ageing-induced systemic inflammation on human skeletal muscle properties [In Press] International Journal of Obesity. Int J Obes (Lond) 2017 ;41(1): 102-111. doi: 10.1038/ ijo.2016.151. Epub 2016 Aug 29.

3. Taylor LA, 2015. Obesity and the Effects of Excess Adiposity on Bone Properties, Health, and Function. Pursuit: The Journal of Undergraduate Research at the University of Tennessee. Vol 6, issue 1. Article 22.

4. Santos, D.A., et al., Sedentary behaviour and physical activity are independently related to functional fitness in older adults. Exp Gerontol 2012; 47(12): p. 908-12.

\section{THE PREVALENCE OF FALLS IN MIDDLE-AGED ADULTS: RESULTS FROM CO-ORDINATED ANALYSES OF HARMONISED DATA FROM FOUR POPULATION-BASED COHORT STUDIES}

Peeters P. ${ }^{1,2}$, van Schoor N.M. ${ }^{3}$, Rachel Cooper R. ${ }^{4}$, Leigh Tooth L. ${ }^{2}$ and Kenny R.A. ${ }^{1,5,6}$

'Global Brain Health Institute, University of California San Francisco, United States of America and Trinity College, Dublin, Ireland; ${ }^{2}$ School of Public Health, The University of Queensland, Brisbane, Australia; ${ }^{3}$ Amsterdam Public Health Research institute, Department of Epidemiology and Biostatistics, VU University Medical Center, Amsterdam, The Netherlands; ${ }^{4}$ MRC Unit for Lifelong Health and Ageing at UCL, London, United Kingdom; ${ }^{5}$ Mercer's Institute for Successful Ageing, St James's Hospital, Dublin, Ireland; ${ }^{6} T$ The Irish Longitudinal Study on Ageing, Trinity College, Dublin, Ireland

Introduction: Onset of declines in balance ability and other measures of physical functioning are typically observed between ages 40 and 60 . Middle-age may be a critical age for early interventions for falls prevention, but the prevalence of falls in this age-range is unknown. The aim was to calculate the prevalence of falls in middle-aged adults (aged 40-64 years) from four countries.

Methodology: Data were from four population-based cohort studies from Australia (ALSWH, n=10556, 100\% women, $51-58$ years in 2004), Ireland (TILDA, $n=4968,57.5 \%$ women, 40-64 years in 2010), the Netherlands (LASA, $\mathrm{n}=862,51.5 \%$ women, $55-64$ years in 2012-13) and Great Britain (NSHD, $n=2821,50.9 \%$ women, 53 years in 1999). Fall status was measured as recall of any falls in the past year. The prevalence of falls was calculated for the total group (unweighted and weighted for age, sex and education), for men and women separately, and for 5-year age-bands.

Results: The average prevalence of falls across the four cohorts was $25.2 \%$, and ranged from $17.5 \%$ in the British cohort to $31.4 \%$ in the Australian cohort. The prevalence was higher in women (26.5\%) than in men $(15.6 \%$, $\mathrm{p}<0.001)$. The prevalence increased from $8.7 \%$ in $40-44$ year olds to $27.8 \%$ in $60-64$ year olds ( $p<0.001$ ), but even within 5-year age-bands, there was substantial variation in prevalence between the four cohorts. Weighting for age, sex and education resulted in estimates that were less than 2 percentage points lower.

Conclusion: A quarter of 40-64 year old adults fall at least once per year. These findings support the notion that falls are not just a problem of old age and warrant further research to inform preventive strategies in middle-aged adults.

\section{OSTEOPOROSIS AND SARCOPENIA IN FEMALE GERIATRIC INPATIENTS ARE INTERRELATED AND ASSOCIATED WITH POOR FUNCTIONAL AND NUTRITIONAL STATE}

Reiter R. ${ }^{1}$, Reiss J. ${ }^{1}$, Kreutzer M. ${ }^{2}$, Weilbuchner I. $^{2}$, Kässmann $\mathrm{H}_{0}{ }^{3}$, Pirich C. ${ }^{3}$ and Iglseder B.

'Department of Geriatric Medicine; '2Department of Clinical Nutrition; ${ }^{3}$ Department of Endocrinology and Nuclear Medicine, Paracelsus Medical University Salzburg, Austria

Introduction: As reflected by the term osteosarcopenia, bone and muscle loss share common causes and reinforce each other. We looked into the degree of overlap between osteoporosis and sarcopenia in female geriatric inpatients from the ongoing SAGE study - a cross-sectional study concerned with methodologic issues of muscle mass measurement.

Methods: So far 51 female SAGE participants (age $80.9 \pm 5.3$ ) underwent measurement of gait speed, hand grip and DXA measurement of muscle mass and bone mineral density. Interrelations between prevalence of sarcopenia (EWGSOP definition based on mass and functionality) and osteoporosis (WHO definition) were explored using Fisher's exact test. Functional (gait speed, handgrip, Barthel index) and nutritional characteristics (MNA-SF, BMI) were compared between sarcopenic/non-sarcopenic and between osteoporotic/non-osteoporotic individuals (t-test/MannWhitney-U-test).

Results: 28 (of 51) women (54.9\%) were neither osteoporotic nor sarcopenic, $12(23.5 \%)$ were only osteoporotic, 2 (3.9\%) were only sarcopenic, and 9 (17.6\%) had both conditions. Sarcopenic individuals had a higher prevalence of osteoporosis compared to non-sarcopenic ( $81.8 \%$ vs. $30.0 \%$; $p<0.005)$. Osteoporotic individuals showed a higher prevalence of sarcopenia $(42.9 \%$ vs. $6.7 \% \%$; $p<0.005$ ).

Sarcopenic women had lower BMI (22.3 vs. $27.3 \mathrm{~kg} / \mathrm{m}^{2}$, $\mathrm{p}=0.002$ ), MNA-SF (8.4 vs. $11.1, \mathrm{p}=0.005$ ), handgrip strength ( 16.2 vs. $21.3 \mathrm{~kg}, p=0.008$ ) and lower femoral neck $(-2.9$ vs. $-1.9, p=0.006)$ and spine $\mathrm{T}$-score $(-2.5$ vs. $-1.1, p=0.007$ ) than non-sarcopenic. Osteoporotic women had lower BMI (23.8 vs. $\left.27.9 \mathrm{~kg} / \mathrm{m}^{2}, p=0.004\right)$, MNASF (9.5 vs. $11.3, p=0.018)$, handgrip strength ( 16.7 vs. 
$22.7 \mathrm{~kg}, \mathrm{p}=0.002$ ) and lower muscle mass index ( $5.5 \mathrm{vs}$. $6.5 \mathrm{~kg} / \mathrm{m}^{2}, \mathrm{p}<0.001$ ) than non-osteoporotic. There were no significant differences in Barthel index and gait speed.

Conclusion: Osteoporosis and sarcopenia showed substantial overlap and both were associated with poorer nutritional state and strength. Osteoporosis and sarcopenia require a concerted diagnostic and therapeutic approach in female geriatric inpatients.

\section{DO OUTCOME MEASUREMENTS IN A FALL PREVENTION SERVICE RELATE TO PATIENT PERCEPTION OF FUNCTIONAL ABILITY? A PILOT SERVICE EVALUATION}

Riley F. and Hood-Moore V.

Nottingham University Hospitals, Nottingham, UK

Introduction: In older persons falls are a significant cause of both disabling physical and emotional decline leading to reduced independence as well as a significant financial burden. Exercise rehabilitation to prevent falls has long been evidenced to be successful. However the studies do not identify changes in a patient's independence. Without improving a patient's functional ability there is a risk of deterioration when rehabilitation ceases.

This evaluation aims to explore whether patient perception of functional change correlates with physical performance measures and psychological questionnaires.

Methods: A mixed methodology service evaluation. Five semi-structured interviews were completed and falls service outcome measurement data collected. Quantitative data analysis was completed within Microsoft Excel calculating the mean of baseline and change. Qualitative thematic analysis was used to analyse and interpret the data. Data were collaborated through case series triangulation.

Results: Only one participant reported significant change in their functional ability, all measures demonstrated improvement but not the highest change in physical outcome measurement.

The change in the berg balance score was not sensitive to change in perceived function ability with the two highest changes in scores denying any change in balance and had altered behaviours adversely to maintain safety.

The most sensitive was the hospital depression and anxiety score with deteriorated scores for all four participants who didn't report significant change in function.

Conclusion: The findings from this evaluation provide important insight in to contextual relationship outcome measurements have with functional ability within the falling population. It could be speculated that physical outcome measurement improvements on their own do not correlate with functional ability. On the basis of this evaluation, a greater emphasis on functional activities is required within the rehabilitation in conjunction with patient orientated goals to ensure functionality is changing.

\section{EXPLORING CHAIR BASED EXERCISE: THE VIEWS OF OLDER PEOPLE AND CARE STAFF}

Robinson K.R. ${ }^{1}$, Leighton P. ${ }^{2}$, Logan P. ${ }^{1}$, Gladman J.R.F. ${ }^{1}$, Harwood R. ${ }^{3}$, Gordon A.L. ${ }^{4}$, Armstrong S. ${ }^{2}$, Anthony K.A. ${ }^{1}$ and Masud T. ${ }^{3}$

'Division of Rehabilitation and Ageing, University of Nottingham; ${ }^{2}$ NIHR Research Design Service for the East Midlands, Faculty of Medicine and Health Sciences; ${ }^{3}$ Healthcare of Older People, Nottingham University Hospitals NHS Trust; ${ }^{4}$ Division of Medical Sciences and Graduate Entry Medicine, University of Nottingham

Introduction: Chair based exercise (CBE) is encouraged for older people with compromised health and mobility and is delivered across health and social care with little standardisation. A CBE programme which consists of moderate intensity aerobic and progressive resistance strength training was developed using a consensus definition. The acceptability of the CBE programme was explored through the views of older people and care staff.

Methodology: Semi-structured interviews were conducted with 8 older people who had participated in the CBE programme and 12 care staff working in care homes, day centres and voluntary groups. Analysis followed the conventions of framework analysis. Data was coded within a pre-defined thematic framework which was structured to consider the perception of $\mathrm{CBE}$, programme content, benefits, as well as barriers and facilitators to participation. The process of analysis followed the stages of coding, charting and summarising.

Results: Older people and care staff found the programme largely acceptable. Benefits primarily focused on mental well-being with physical health benefits not commonly discussed. Barriers to participation focused around poor health and limited physical abilities which was consistently reported by older people and care staff. There appeared to be a conflict between some staff who felt CBE was the only exercise older people could do and some older people who felt they wanted to try more 'proper' standing exercise, although some were not confident they would have been able to undertake it.

Conclusions: Older people and care staff primarily view CBE as an intervention to support mental well-being with few physical health benefits reported. If CBE is to be used for the purpose of mental well-being the method of evaluation needs to reflect this purpose. Programmes that support the progression to standing and walking exercises should be explored within these settings.

\section{OUTCOME OF FALLS INCIDENCE FOLLOWING IMPLEMENTATION OF A FALLS PREVENTION PROGRAMME IN A RESIDENTIAL CARE SETTING}

Rodger D., Hussey P. and Murphy C.

St Mary's Hospital \& Dublin City University, Ireland. 
Introduction: Falls and falls related injuries are the second leading cause of accidental or unintentional injury deaths worldwide with an estimated 424000 people dying each year as a result of a fall (WHO, 2016). Statistics show one in three people over 65 years and one in two people over 80 years fall each year with up to $15 \%$ having a subsequent fall within six months. The age profile of older adults living in residential care settings is rising with the average age being 84 years. Up to $50 \%$ of older people in residential care facilities fall at least once a year and up to $40 \%$ will have a subsequent fall. $8 \%$ will have a hip fracture (IHFD, 2016) Hip fractures have been identified as one of the most devastating injuries for older people who fall (DoH, HSE 2008) resulting in increased mortality and morbidity. Measures to reduce the risk of falls in this vulnerable population are necessary.

Methodology: Audit of falls in a residential care facility identified the need for a review of falls prevention strategies to reduce the number of and raise awareness of falls. An action research project commenced in 2011 to develop and implement a falls prevention programme. This programme included the development of a supporting education resource.

Results: In the first year post implementation there was a $35 \%$ reduction in falls across the facility when compared to pre programme data in the year before. Year on year since there is a sustained reduction in falls.

Conclusion: This study demonstrates that falls prevention programmes have the potential to reduce the risk of falls in residential care settings. However further studies are required to establish what causes the sustained reduction. A case study on the action research programme is now in progress and will be reported upon further in the presentation.

\section{BONE HEALTH IN INTELLECTUAL DISABILITY POPULATION: EMPOWERMENT OF PEERS THROUGH EDUCATION}

\section{Rodger D., Ledger L. and Spencer A.}

St Mary's Hospital \& St Michaels House, Dublin, Ireland

Introduction: The Irish LongituDinal DA on Ageing (TILDA) Intellectual Disabilities Study Wave (2014) report osteoporosis and falls related injuries are a significant health concern for people with an intellectual disability, with $70 \%$ having reduced bone density and 33\% of those aged over 50 years falling each year. Despite these numbers there was no education resource to inform health and social care staff, families, carers and people with an intellectual disability of these facts and on measures required for their prevention and management.

Methodology: In 2014 Forever Autumn Community of Practice (FACOP) collaborated with intellectual Disability (ID) service providers to develop an education resource on bone health and falls awareness. A multidisciplinary approach involving service users informed the content. The aim of the education resource is to provide information on bone health and falls awareness with a specific focus on exercise and nutrition for people with an ID. It also aims to raise awareness of reduced bone mineral density and risk of falling in this population.

Results: Through engagement with an Educational Technologist to design and develop the content, a suite of resources is available on an open online platform called Happy Bones. Service users and their families were actively involved in the development of the educational resources. The material is developed in a format that makes it easily transferable - on line through website, eBook store, and YouTube - including 3 short videos in which service users feature - Paula's Story, Having a DXA scan, and Best Foot Forward - Chrissies Story.

Conclusion: Happy Bones has generated a lot of interest both nationally and internationally. The programme has resulted in an increased awareness of the importance of good bone health, falls awareness and falls prevention strategies among all age groups.

Through their new knowledge the service users initiated the promotion of the resources at many events within their organisation and further afield. This facilitated sharing of their learned experiences with their peers to promote a healthy active lifestyle and to achieve strong bones. It demonstrates how this collaboration across service providers supporting each other can be achieved.

\section{ACTIVITIES OF DAILY LIVING AT ADMISSION TO ACUTE GERIATRIC DEPARTMENTS PREDICTS HIP FRACTURE: A NATIONWIDE COHORT STUDY}

Ryg J. ${ }^{1}$, Engberg H. ${ }^{2}$, Hansen H.H. ${ }^{2}$, Mariadas P. ${ }^{2}$, Pedersen S.G.H. ${ }^{3}$, Jorgensen, M.G. ${ }^{4}$, Vinding K.L. ${ }^{5}$ and Andersen-Ranberg K. ${ }^{1}$

'Department of Geriatric Medicine, Odense University Hospital, Denmark (DK); ${ }^{2}$ Center for Clinical Epidemiology, Odense University Hospital, DK; ${ }^{3}$ Medical Department, Holbæk Hospital, DK; ${ }^{4}$ Department of Geriatrics, Aalborg University Hospital, DK; ${ }^{5}$ Medical Department, Kolding Hospital, DK

Introduction: Barthel Index-100 (BI) is used to measure geriatric patients' activities of daily living. We studied whether routine $\mathrm{BI}$ assessment at time of hospital admission predicts future hip fracture (HFx).

Methods: This population-based cohort study included all patients aged $\geq 65$ years from the National Danish Geriatric Database during 2005-2014. Patients were followed until HFx, death, or end of study. BI was assessed at admission to a geriatric department and linked at the individual level to Danish national health registers. BI-score was reported numeric and categorized in four standard sub-categories $(\mathrm{Bl}=0-24, \mathrm{Bl}=25-49, \mathrm{Bl}=50-79, \mathrm{Bl}=80-100)$. Fine and Gray's competing-risks regression model was used to estimate the cumulative fracture incidence and hazard ratios 
adjusting for relevant confounders (i.e. age, civil status, BMI, Charlson Comorbidity Index, polypharmacy, prior fracture).

Results: Totally 74,603 patients were included. Women $(63 \%)$ were significantly older and had higher BI than men ((median [IQR]) 84[79-89] vs. 81[76-86] years; BI (55[(30-77] vs. 52[26-77]). Totally 27,689 (37.1\%) had prior major osteoporotic fracture. At admission 9,155 $(12.3 \%)$ were in anti-osteoporotic treatment. Median follow-up was 1.8 years (range 0-11 years), corresponding to 178,836 person years (py). A total of $7,296(9.8 \%)$ had a HFx during follow-up. The incidence of HFx was 45/1,000 py in women and $32 / 1,000$ py in men. The cumulative 2 -year incidence of $\mathrm{HFx}$ was $6.4 \%$ for the overall cohort being highest in sub-category $\mathrm{BI}=25-49$ for women $(8.3 \%)$ and men (5.6\%). In the multivariate analysis using $\mathrm{BI}=80-$ 100 as reference the adjusted hazard ratio for $\mathrm{HFx}(95 \% \mathrm{Cl})$ in women and men was 0.82(0.72-0.94) and 1.07(0.861.32) for $\mathrm{Bl}=0-24,1.17(1.05-1.31)$ and $1.40(1.15-1.70)$ for $\mathrm{Bl}=25-49$, and $1.15(1.04-1.28)$ and $1.41(1.18-1.70)$ for $\mathrm{Bl}=50-79$, respectively.

Conclusion: $A D L$ is an independent predictor of future hip fracture following admission to geriatric department. We propose the need for increased fracture prevention awareness in these patients.

\section{DEVELOPING A PHYSICAL FITNESS AND BLADDER HEALTH INTERVENTION TO REDUCE THE RISK OF FALLS AMONG OLDER COMMUNITY-LIVING ADULTS}

Booth L. ${ }^{1}$, Skelton D.A. ${ }^{1}$, Hagen S. ${ }^{2}$ and Booth J. ${ }^{1}$

"School of Health and Life Sciences, Institute of Allied Health Research, Glasgow Caledonian University, UK, ${ }^{2}$ NMAHP RU, Glasgow Caledonian University, UK

Introduction: Falls, frailty, poor physical function and urinary incontinence $(\mathrm{UI})$ are associated and both contribute and are caused by an inactive lifestyle ${ }^{1}$. The Functional Fitness MOT $(\mathrm{FFMOT})^{2}$ is an intervention to promote physical functioning in community-living older adults. The FFMOT focuses on components of strength and balance, important factors for reducing the risk of falls. Ul awareness is not currently part of the FFMOT. Developing a combined intervention to improve physical functioning and self-management of bladder health has the potential to further reduce the risk of falls.

Methodology: Our aim is to develop the FFMOT to include bladder health promotion. This abstract reports the first phase of this work. A systematic literature review was performed to identify a bladder symptom screening tool that was valid, reliable and brief, for addition to the current FFMOT. We also analysed a large existing dataset to develop normative data scores for the identified screening tool.

Results: Systematic Review: 19 studies were included in the review. The International Prostate Symptom Score (IPSS) is a short (7-symptom items, 1-quality of life item), self- completion bladder-health screening questionnaire, found to have good validity and reliability scores for men and women. Normative Data Study: Data from 503 men and 579 women living in the UK, aged between 60 and 99 years, were analysed. Median scores and $25^{\text {th }}$ to $75^{\text {th }}$ percentile scores indicate that normal ranges of IPSS score are mild (0-7) or moderate (8-19) in severity, irrespective of age or gender.

Conclusion: For ease of use and trustworthiness, the IPSS is recommended for use within combined health promotion interventions. These first IPSS normative values for a representative sample of healthy community-living older adults in the UK will be useful for researchers and practitioners to allow individual IPSS scores for comparison with peers of similar age and gender.

\section{References}

1. Gibson $W$ et al. The association between lower urinary tract symptoms and falls: Forming a theoretical model for a research agenda. Neurourol Urodyn 2017. doi: 10.1002/nau.23295.

2. de Jong LD, et al. The Functional Fitness MOT Test Battery for Older Adults: Protocol for a Mixed-Method Feasibility Study. JMIR Res Protoc 2016:5(2):e 108.

\section{MAKING REASONABLE ADJUSTMENTS TO PROVIDE EQUITABLE OSTEOPOROSIS ASSESSMENT, SCREENING AND MANAGEMENT FOR PEOPLE WITH LEARNING DISABILITIES: INTERIM RESULTS}

De Amicis L., Finlayson J., Skelton D.A., Gallacher S., Munro R., Crockett J. and Godwin J.

School of Health and Life Sciences, Glasgow Caledonian University, Cowcaddens Road, Glasgow G4 OBA

Introduction: People with learning disabilities (LDs) are athigh risk of developing osteoporosis, due to a higher prevalence of factors which are more relevant to this population; having Down's syndrome, immobility, and being prescribed antiepileptic drugs. People with LDs can experience barriers to accessing appropriate health care e.g. lack of access for wheelchair users. The UK Equality Act 2010 requires health services to make reasonable adjustments to ensure equity of access for people with disabilities.

Method: This feasibility study is identifying and implementing reasonable adjustments with 30 adults with LDs and one or more risk factor, to conduct osteoporosis assessment, DXA screening and management (where applicable) across two hospital sites in Scotland.

Results: Interim analysis of 10 participants with LDs who have completed DXA bone scans are as follows: average age of participants is 43 years (ranging from 26 to 62 years); 6 are male; and 6 have been identified as having osteopenia/ osteoporosis post-assessment. Of the ten, 4 have Down's syndrome, 1 is immobile, and 6 are currently prescribed anti-epileptic drugs. All ten benefited from at least one reasonable adjustment, and new reasonable adjustments not 
previously reported for people with LDs have been identified e.g. preferring the hospital appointment to be scheduled around the person's daily routine to lessen anxiety.

Conclusion: People with LDs require tailored osteoporosis assessment and screening, due to these specific risk factors being experienced by both women and men with LDs at any age. Reasonable adjustments are identified and recommended for patients with LDs.

\section{PERSON-CENTRED MOVING AND HANDLING IN OLDER PEOPLE WITH OSTEOPOROSIS: AN ACTION RESEARCH AND PROCESS EVALUATION STUDY}

Smith M.A.C., Pearson C., Combe K., Bacigalupo A. and McCormack B.

Division of Nursing, School of Health Sciences, Queen Margaret University, Edinburgh, EH2 $16 \mathrm{UU}$

Introduction: Osteoporosis is a highly prevalent bone disease world-wide that significantly increases fracture risk ${ }^{5}$, patient morbidity and mortality ${ }^{3}$. Osteoporosis increases with age and hip fractures will be a major public health issue by $2050^{\prime}$. Implications for nursing care, particularly moving and handling are under-researched. Falls risk screening is widely implemented, but fragility fracture screening less so. Moving and handling can be complex in older people with osteoporosis ${ }^{2}$. Nurses' perspectives on moving and handling require exploration 4 .

Methods: A participatory action research and process evaluation design addressed contextual factors and implemented an intervention to promote person-centred moving and handling in acute care. Research questions focused on staff experiences of a complex education intervention, views on safe and effective moving and handling, and older people's experiences of moving and handling. Phase 1 adapted an education intervention from the Lydia Osteoporosis Project to the local context using coproduction methods with a purposive sample of NHS staff. Phase 2; process evaluation, elicited views of staff about osteoporosis, experiences of an online module and action research, and patients' experiences. Data collection methods included focus groups, site visits, online surveys, interviews. Codes and categories were applied to qualitative data, analytic themes were identified and descriptive quantitative analysis of survey data performed.

Results: Staff participants $(\mathrm{N}=33)$ viewed Osteoporosis as complex, and reported an increased understanding of implications for safe and effective moving and handling practice in this context. Online learning was viewed positively. Experiences of people in receipt of care were elicited.

Conclusions: Use of action research \& process evaluation encouraged active engagement of frontline staff in learning about Osteoporosis and fracture risk. Implications for practice, a person-centred approach to moving and handling and perspectives from persons in receipt of care were identified.

\section{References}

1. Cooper C, Campion G, Melton LJ. Hip fractures in the elderly: a worldwide projection. Osteoporosis Int 1992;2(6):285-9

2. Coulter Smith MA 2016a, O’ May F. Tropea S, Berg J Framing moving and handling as a complex intervention in the acute care of older adults with osteoporosis: A qualitative study. Journal of Clinical Nursing 25, 2906-2920, doi: 10.1111/jocn.13344 Wiley Open Access.

3. IOF 2017 International Osteoporosis Foundation. Osteoporosis Facts and Statistics. http://www.iofbonehealth.org/facts-statistics Accessed 29.06.17

4. Kay K, Evans A \& Glass N. Moments of speaking and silencing: nurses share their experiences of manual handling in healthcare. Collegian 2015;22:61-70

5. SIGN 2015 Management of osteoporosis and the prevention of fragility fractures Guideline No.142, ISBN 9781909103351 . Published March 2015, Scottish Intercollegiate Guidelines Network, Health Improvement Scotland http://sign.ac.uk/guidelines/ fulltext/142/index.html Accessed 29.06.17

6. Lydia Osteoporosis Final Report. Coulter Smith M A, Pearson C, O'May F, Tropea S, Irvine L, Rush R, Wilson R. 2016b Final Project Report for the Lydia Osteoporosis Project, completed May 2016. http://eresearch.qmu.ac.uk/4419/1/4419.pdf

\section{“OFF LEGS" - LOOK WELL BEYOND THE LEGS. A CLINICAL CASE STUDY}

Tay H.S. and Qureshi A.

Department of Geriatric Medicine, Aberdeen Royal Infirmary. NHS Grampian. UK

Case Study: A 76 year old male ex-smoker was referred with a short history of impaired mobility and falls. He had also developed urinary incontinence and mild cognitive impairment.

His blood pressure was normal with no postural drop. Power was slightly weak in the legs but reflexes and sensation were normal. His gait was ataxic and cognition was mildly impaired.

The clinical impression was of a possible cerebrovascular event, or normal pressure hydrocephalus. A computer tomography (CT) head scan showed a brain stem mass causing hydrocephalus. A cerebrospinal fluid (CSF) flowstudy confirmed obstruction at the level of the tumour. CT chest and abdomen was normal.

A diagnosis of obstructive hydrocephalus was made and a neurosurgical opinion sought. Tumour location precluded resection or biopsy, but CSF did not show any malignant cells. A ventriculo-peritoneal shunt was inserted and he was commenced on a trial of dexamethasone to cover the possibility of cerebral lymphoma. A subsequent CT scan showed ventricular reduction but no change in tumour size. His mobility has now improved and he is no longer incontinent or confused.

Discussion: Obstructive hydrocephalus occurs when there is a block in free drainage between the ventricles. Such obstruction can lie between the lateral and third ventricle, or the narrow passage connecting the third and fourth 
ventricles - the cerebral aqueduct (of Sylvius). This channel lies in the midbrain and obstruction may be due to pressure effects from primary or secondary tumours.

Normal Pressure Hydrocephalus is a form of non-obstructive hydrocephalus. This classically presents with Hakim's triad: gait instability, urinary incontinence and dementia. All features were present to some degree in this case. However this condition is controversial, and also difficult to diagnose in older people in whom co-incident ventricular dilatation due to cerebral atrophy is highly prevalent.

\section{THE ASSOCIATION OF THE CLINICAL FRAILTY SCALE WITH HOSPITAL OUTCOMES IN PARKINSON'S DISEASE PATIENTS}

Torsney K.M. and Romero-Ortuno R.

Clinical Gerontology Unit, Department of Public Health and Primary Care, University of Cambridge, UK

Introduction: Parkinson's disease (PD) and frailty are both common conditions affecting older people. Little is known regarding the association of the clinical frailty scale (CFS) with hospital outcomes in idiopathic PD patients admitted to the acute hospital. Our aim was to address this knowledge gap.

Methods: We conducted an observational retrospective study in a large tertiary university hospital. Routinely measured patient characteristics included demographics (age and sex), CFS, acute illness severity (Emergency Department modified Early Warning Score, ED-MEWS), the Charlson Comorbidity Index (CCl), discharge specialty, history of dementia, history of depression and the presence of delirium. Outcomes studied were inpatient mortality, death within 30 days of discharge, new institutionalisation, length of stay (LOS) $>7$ days and 30-day readmission rate.

Results: Between October 2014 and October 2016, there were 393 first admission episodes of idiopathic PD patients aged 75 years or more, of which $42.2 \%$ were female. The mean age (SD) was 82.8 (5.0) years. The mean CFS (SD) was 5.9 (1.4) and the mean CCl (SD) was 1.3 (1.5). After adjustment for covariates, CFS was an independent predictor of inpatient mortality with an odds ratio (OR) of 2.0, 95\% $\mathrm{Cl}$ 1.3-3.0, $\mathrm{p}<0.001$. The other significant independent predictor of inpatient mortality was ED-MEWS (OR 1.3, 95\% $\mathrm{Cl} 1.0-1.6, p=0.03$ ). The CFS also predicted death within 30 days of discharge (OR $1.8,95 \% \mathrm{Cl} 1.1-2.8, \mathrm{p}=0.02$ ). It did not significantly predict any of the other outcomes.

Conclusions: The CFS was a significant predictor of inpatient mortality and mortality within 30 days of discharge in idiopathic PD patients admitted to the acute hospital and it might be useful as a marker of risk in this vulnerable population.
EVALUATION OF NUTRITIONAL STATE OF THE ELDERLY OVER 60 YEARS WITH CHRONIC DISEAES IN THE PALLIATIVE CARE CLINIC OF THE WALTER FERRARI HOSPITAL, JAGUARIUNA, SAO PAULO, BRAZIL

Bestane G., da Silva F.T., Soares M.M., Bacha D.G., Sasaki J. and Tourinho M.L.

Walter Ferreri Hospital - City of Jaguariuna, Sao Paulo, Brazil

Introduction: Aging individual is regarded as a physiological process that subjects the body to various functional, metabolic and anatomical changes affecting the body generally. In elderly patients, the weakness is a condition characterized by reduced physiological reserves leading the body to vulnerabilities that can lead to weight loss, sarcopenia, decreased strength and mobility, slow, physical inactivity and low energy expenditure. The nutritional status of the elderly is a determining factor in the quality of life and mortality of these patients. The objective was to know the nutritional status of the elderly over 60 years with chronic diseases seen in the ambulatory palliative care Municipal Hospital Walter Ferrari, Jaguariúna, Sao Paulo.

Methods: Field research, quantitative and descriptive was carried out, we used full clinical examination, laboratory tests and a questionnaire including MAN to evaluate the elderly in a normal nutritional status (MAN: 24-30), nutritional risk (MAN: 17-23 5) and malnourished (MAN: <17.0). We evaluated 50 patients with chronic diseases and established correlations between clinical, biochemical metrics and ratings PPS with the result obtained by MAN methodology.

Results: The elderly had altered nutritional tables due to physiological and metabolic disorders use of drugs that contribute to non-absorption of nutrients associated with chronic diseases that worsen the rames of fragility. Malnutrition was considerable precedent of disease and death in the elderly. The assessment carried out by MAN method showed that there was a direct link between the presence of chronic diseases and the deterioration of nutritional status. Anthropometric tools are a good indicator of nutritional status. Seniors, who had regular measures of calf and arm, mostly presented regular framework of nutrition according to the methodology. Another marker that proved reliable and establishing correlation as MAN method is serum albumin. Individuals with low albumin levels obtained low scores also in methodology factors directly related to nutrition and metabolic and physiological changes, as well as medicines that impair nutrient absorption should also be considered when assessed the nutritional status of the individual.

Conclusion: A healthy life, social life and adequate food contribute to the longevity of elderly patients with chronic diseases bringing quality of life and maintenance of autonomy and individual functionality. 


\section{EVALUATION OF QUALITY OF LIFE ON PATIENTS IN THE PALLIATIVE CARE CLINIC OF THE WALTER FERRARI HOSPITAL, JAGUARIUNA, SAO PAULO, BRAZIL}

\author{
da Silva F.T., Bestane G., Soares M.M., Bacha D.G., Sasaki \\ J. and Tourinho M.L. \\ Walter Ferreri Hospital - City of Jaguariuna, Sao Paulo, Brazil
}

Introduction: The ageing of the individual is as a physiological process and the elderly population is quickly growing in Brazil. Health is a basic need of every human being. In recent decades, medicine has been directing a broader view, in which the patient outside therapeutic possibilities of cure, receive care in order to provide quality of life throughout the disease process and its final moments. The objective of this study was to analyze the quality of life of patients with serious, progressive and incurable diseases treated at palliative care service at the Hospital Walter Ferrari.

Methods: A research of quality of life of patients in the Palliative Care Clinic was performed using clinical data and a standard questionnaire abbreviated Woqol.

Results: 50 patients were evaluated with chronic diseases, aged 21-97 years. It was possible to observe that patients were mostly female, suffering from chronic diseases, there is no need (for the most part of the sample) of care exercised by caregivers.

Participants in the study were not satisfied with the quality of life they present, however, the vast majority reported being satisfied with the health services that they receive. The Palliative Performance Scale - PPS ranged from 10\% to $100 \%$.

\section{THE PROMOTING ACTIVITY, INDEPENDENCE AND STABILITY IN EARLY DEMENTIA (PRAISED) FEASIBILITY STUDY - BASELINE DATA}

van der Wardt V. ${ }^{1}$, Goldberg S. ${ }^{2}$ and Harwood R.H. ${ }^{3}$

'Division of Rehabilitation and Ageing, School of Medicine; University of Nottingham; '2School of Health Sciences, University of Nottingham; ${ }^{3}$ Nottingham University Hospitals NHS Trust. UK

Introduction: People with dementia are at high risk of falls with significant costs to the individual, their relatives and society. The PrAISED study evaluates a multi-component intervention including physiotherapy, functional activities and motivational psychology approaches to support people with mild Dementia to stay independent for longer. The purpose of the feasibility study was to evaluate recruitment rates, procedures and intervention implementation. All baseline assessments were completed in March 2017.

Methodology: The feasibility RCT included two groups using different intensity interventions (50 visits over one year and 9 visits over 3 months) and a control group. Each group included 20 participants recruited from memory clinics and Join Dementia Research (JDR; a register of people interested in dementia research). Participants were assessed at baseline, follow-up is at 12 months. The main outcome is independence (Disability Assessment for Dementia (DAD) and Nottingham Extended Activity of Daily Living (NEADL)), secondary outcomes included falls, cognition, frailty, quality of life, depression, activity levels, attitudes to exercise, mobility and health service use.

Results: Recruitment for this feasibility study was completed on target. The baseline assessments were long (up to 3.5 hours) and 13 out of 60 participants experienced them as burdensome. An initial analysis of the baseline data showed that participants had a mean age of 76 years (SD 6.9) and included $57 \%$ men. The DAD was completed by $87 \%$ of participants ( $n=6$ missing to due absence of informant), the mean DAD score was 77.9 (SD 21.3), the mean NEADL score 16.3 (SD 4.4) and the mean SMMSE score 25.6 (SD 3.1). Further data will be presented at the conference.

Conclusion: A combination of recruitment via Memory Clinics and JDR ensured successful recruitment. Baseline assessment duration will be decreased for the main trial and the availability informants will be included as eligibility criterion.

\section{POST DISCHARGE HOSPITAL READMISSION IN A COHORT OF HIP FRACTURE PATIENTS}

Kershenbaum A. and Vindlacheruvu M.

Orthogeriatric Department, Cambridge University Hospitals NHS Foundation Trust, Addenbrookes Hospital, Hills Road, Cambridge, UK

Introduction: Apart from measurement of $\mathbf{3 0}$ day mortality, post discharge progress is not routinely examined.

We examined a cohort of hip fracture patients to assess risk of readmission and cause of readmission.

Methods: We followed a cohort of 393 patients age 60 or over admitted to Addenbrookes (March 2015 - February 2016) with a hip fracture. Using the National Hip Fracture Database (NHFD) and computerised hospital records we measured time to readmission from discharge and time from admission to death. We used Kaplan-Meir curves to examine survival and readmission over time. The first readmission episode only was considered and elective admissions excluded.

We divided the cohort by age, abbreviated mental test score (AMTS) at admission, mobility pre-admission, residence type at admission, American Society of Anesthesiologists (ASA) Score and examined readmission by subgroups. We identified cause of readmission by review of the medical records.

Results: Of 393 patients, 74 died ( $18.8 \%$ ) within 180 days from admission. 13 patients died during the admission or on the day of discharge (3.3\%).

Of 380 patients who were discharged, 133 were readmitted (35.0\%) within 180 days from discharge. The rate of readmission was particularly high close to discharge. 
Markers of frailty including lower AMTS, higher age at admission, poorer mobility pre-admission, nursing/ residential home/hospital residence pre-admission and higher ASA score were each associated with a significantly increased risk of readmission.

The most common cause of readmission was fall/collapse in $47 / 133(35.3 \%)$ patients, followed by chest infection in $33 / 133$ (24.8\%) where 3 cases recorded both causes.

Conclusion: Patients recently admitted with a hip fracture are at high risk of a repeated readmission often due to a repeated episode of fall/collapse. Those patients with a marker of frailty were at increased risk. Interventions to reduce the risk of a repeated fall might improve post-discharge progress in this frail population.

\section{A RANDOMISED CONTROLLED TRIAL AND ECONOMIC EVALUATION OF A COMMUNITY-BASED PHYSICAL ACTIVITY INTERVENTION TO PREVENT MOBILITY-RELATED DISABILITY IN OLDER ADULTS. THE REACT (RETIREMENT IN ACTION) STUDY}

Stathi A. ${ }^{1}$, Withall J. ${ }^{1}$, Greaves C.J.2, Thompson J.L. ${ }^{3}$, Medina-Lara A. ${ }^{2}$, Taylor G. ${ }^{1}$, Bilzon J. ${ }^{1}$, Gray S. ${ }^{5}$, Green C. ${ }^{2}$, Johanssen-Berg H. ${ }^{6}$, Sexton C. ${ }^{6}$, Demnitz N. ${ }^{6}$, De Koning J. ${ }^{1}$, Western M. ${ }^{1}$, Bollen J. ${ }^{2}$, Moorlock S. ${ }^{3}$ and Fox K.R. ${ }^{4}$ 'University of Bath UK; ${ }^{2}$ University of Exeter UK; ${ }^{3}$ University of Birmingham UK; ${ }^{4}$ University of Bristol UK; ${ }^{5}$ University of West of England UK; ${ }^{U}$ University of Oxford UK

Introduction: During old age, there is a population-wide transition from independence and adequate physical function towards frailty, mobility-related disability and increased demand for health and support services. The REACT study assesses an intervention designed to challenge this progression.

Methods: A multi-centre, pragmatic, randomised controlled trial with an internal pilot phase and followup of 24 months. Participants: 768 sedentary people aged 65 and over at risk of major mobility limitations but still ambulatory. Primary outcome: Functional ability measured by the Short Physical Performance Battery at 24 months. REACT Intervention: A 12-month programme delivered in leisure/community/fitness/health centres in Bath, Bristol, Birmingham and Devon to provide economic and ethnic diversity. REACT targets strength, flexibility, lower-limb function and balance, includes social and education sessions, and is delivered in two progressive phases. Established behaviour change tech niques are used to enhance motivation, overcome barriers and facilitate lifestyle changes. fMRI substudy: Structural and functional brain MRI measures for a sub-sample of participants via a 3T MRI scan. Detailed spatio-temporal measures of gait test associations between mobility and cognition. Process evaluation: The quality and quantity of intervention delivery, the proposed mechanisms of change and feasibility of implementation are evaluated via mixedmethods assessment.

Results: REACT assesses the effectiveness and costeffectiveness of a community-based physical activity intervention for reducing the progression of functional limitations in older adults at high risk for mobility-related disability. The MRI substudy tests whether a physical exercise intervention slows the rate of brain atrophy and decline in cognitive function.

Conclusions: The REACT study is a complex intervention which, if effective and cost-effective, could lead to significant public health impact by preventing frailty and tackling inactivity in older adults.

\section{A SYSTEMATIC REVIEW OF PODIATRY INTERVENTIONS TO REDUCE FALLS IN OLDER PEOPLE}

Wylie G. ${ }^{1}$, Torrens C. ${ }^{2}$, Campbell P. ${ }^{2}$, Frost H. ${ }^{3}$, Witham M. ${ }^{4}$ and Morris J.4

'University of Dundee/ NHS Tayside; ' ${ }^{2}$ MAHP Research Unit, Glasgow Caledonian University; ${ }^{3}$ University of Stirling; ${ }^{4}$ University of Dundee

Introduction: Evidence for foot and footwear problems as a contributing factor for falls in older people is well established. However, the effectiveness of podiatry interventions in addressing these problems, to reduce falls, has not been established. We systematically reviewed evidence for the effectiveness of podiatry interventions to reduce falls in older people living in care homes or the community.

Methods: We systematically searched MEDLINE, AMED, EMBASE, CINAHL, PEDro, Cochrane Central Register of Controlled Trials, CDSR, DARE, HTA, ZETOC, WHO ICTRP, EThOS and Google Scholar (from inception to 30 March 2017, with no language restrictions). We hand-searched reference lists and contacted experts. We included all randomised controlled trials (RCT) or quasi-RCT studies that documented podiatry interventions in older people (aged 60+). Two reviewers independently applied selection criteria and assessed methodological quality using the Cochrane risk of bias tool. Data were extracted in accordance with TiDieR guidelines and tabulated and summarised in a narrative format.

Results: We identified 32717 titles and screened 3118 abstracts for eligibility. Seven studies ( 14 reports) met our inclusion criteria, trials generally were judged as potentially high quality. Total sample size was 2732 , range $40-1010$ participants. Most podiatry interventions were multi-faceted although 2 were single component interventions (e.g. insoles or footwear). The majority of interventions were conducted with older people in the community $(n=5)$ Delivery was faceto-face and by telephone $(n=2)$. Five trials reported beneficial effects on falls rate $(n=1)$ and falls risk (e.g. proportion of fallers, balance, foot pain) $(n=4)$. 
Conclusions: This is the first study to synthesise the available evidence of podiatry interventions for falls prevention in older people living in care homes or in the community. The evidence is limited, in particular in care homes, however there is some indication that that multifaceted interventions have the potential to be successful in falls prevention.

\section{REDUCING FALLS RISK WITH KINECT BASED FALLS PREVENTION EXERGAMES: A 12 WEEK TWO- CENTRE, CLUSTER RCT OF COMMUNITY-DWELLING OLDER ADULTS LIVING IN SHELTERED HOUSING}

Stanmore E. ${ }^{1}$, Mavroeidi A. ${ }^{2}$, Bell V. ${ }^{1}$, Meekes W. ${ }^{1}$, de Jong L.D. ${ }^{3}$, Skelton D.A. ${ }^{2}$, Sutton C.J. ${ }^{4}$, Benedetto V. ${ }^{4}$ and Todd $\mathrm{C}^{1}$

'University of Manchester, School of Health Sciences, Division of Nursing, Midwifery and Social Work, UK, ${ }^{2}$ School of Health and Life Sciences, Institute of Allied Health Research, Glasgow Caledonian University, UK, ${ }^{3}$ School of Physiotherapy and Exercise Science, Faculty of Health Sciences, Curtin University, Perth, Australia, ${ }^{4}$ Lancashire Clinical Trials Unit, Faculty of Health and Wellbeing, University of Central Lancashire, UK

Introduction: Falls are the leading cause of fatal and non-fatal unintentional injuries in older people. Despite overwhelming evidence for strength/balance training, adherence to both group and home-exercise programmes is often poor ${ }^{1}$. A novel approach is the use of home-based, strength and balance video-games (EXERGAMES) but data from randomized controlled trials are limited ${ }^{2}$. The aim of this study was to test whether a 12 week EXERGAMES home-based intervention, co-developed with older adults and therapists, can improve the risk of falling [by Berg Balance Scale (BBS)] in older adults living in sheltered housing.
Methods: We conducted a two-site (Manchester/Glasgow) cluster RCT. Twelve sheltered housing facilities were randomised $(1: 1)$ to either a $12 \mathrm{wk}$ standard care plus 3 times/ week EXERGAMES physiotherapist one-to-one intervention $(n=56)$ or standard care (control group $n=50)$. Standard care involved the provision of a home exercise booklet (standard approach for those unable/unwilling to attend group exercise programmes in the community).

Results: A total of 106 older adults ( $83 \mathrm{~F}, 23 \mathrm{M})$ with a mean age of $77.8 \mathrm{y}$ (SD 10.2; range 55-101y), were recruited for the study.

A total of 91 (86\%) participants completed all study visits. Over 12 weeks the EXERGAMES intervention had a significant positive impact on BBS [ $p=0.003$; mean (SD), 44.6 (10.7) (EXERGAMES) vs. 37.6 (14.9) (Control)]. The mean change of BBS from baseline was 2.9 (8.5) for the EXERGAMES and -2.8 (6.5) for the control group. The estimated increase in BBS score due to EXERGAMES was 6.2 (95\% $\mathrm{Cl} 2.4$ to 9.9) which is greater than the minimal detectable change for the scale of $4.9^{3}$.

Conclusions: Balance, and thus risk of falling, can be improved through a 12 wk physiotherapy led EXERGAMES programme. This type of home based intervention could be considered as an effective alternative to traditional falls prevention exercise regimes to support outcomes for patients.

\section{References}

1. Nyman, S.R. and C.R. Victor, Older people's participation in and engagement with falls prevention interventions in community settings: an augment to the Cochrane systematic review. Age Ageing 2012;41(1): 16-23.

2. Skjaeret $N$, et al. Exercise and rehabilitation delivered through exergames in older adults: An integrative review of technologies, safety and efficacy. Int J Med Inform 2016;85(1): 1-16.

3. Donoghue, D., et al., How much change is true change? The minimum detectable change of the Berg Balance Scale in elderly people. J Rehabil Med 2009;41(5):343-6. 